\title{
On the vorticity characteristics of lobe-forced mixer at different configurations
}

\author{
R. Mao $\cdot$ S. C. M. Yu $\cdot$ T. Zhou $\cdot$ L. P. Chua
}

Received: 22 January 2008/Revised: 24 December 2008/Accepted: 31 December 2008/Published online: 25 January 2009

(C) Springer-Verlag 2009

\begin{abstract}
Lobe-forced mixer is one typical example of the passive flow controllers owing to its corrugated trailing edge. Besides the spanwise Kelvin-Helmholtz vortex shedding, streamwise vortices are also generated within its mixing layer. The geometrical configuration of the lobe significantly affects these two types of vortices, which in turn affects the mixing performance of the mixer. In the present investigation, characteristics of mixers with five different configurations were examined and evaluated for two velocity ratios $(r=1,0.4)$. The mixers have only one lobe in order to eliminate any possible interactions between neighboring vortices generated by the adjacent lobes. Hotwire anemometer was used to examine the Kelvin-Helmholtz vortices via the spectrum analysis while laser Doppler anemometer was employed to examine the streamwise vortices. It was found that there were two main frequencies for the Kelvin-Helmholtz vortices in the wake of the mixer; and the Strouhal numbers approached their respective maximum values at high Reynolds number. The rectangular mixer had similar mixing performance with the semicircular one; and both of them were better than the triangular mixer. The scalloping modification enhanced
\end{abstract}

R. Mao $(\square)$

Faculty of Civil Engineering, Delft University of Technology,

Delft, The Netherlands

e-mail: r.mao@tudelft.nl

S. C. M. Yu · L. P. Chua

School of Mechanical and Aerospace Engineering,

Nanyang Technological University, Singapore,

Republic of Singapore

T. Zhou

School of Civil and Resource Engineering,

University of Western Australia, Crawley, Australia

mixing by generating additional streamwise vortices while the scarfing modification could not improve the mixing performance.

\section{List of symbols}

$A_{\text {wake }} \quad$ Wake area of the lobe-forced mixer flow $\left(\mathrm{m}^{2}\right)$ $C_{\mathrm{I}} \quad$ Normalized streamwise circulation $C_{\mathrm{I}}=\frac{\Gamma_{I}}{\bar{U} \times \lambda}$

$R \quad$ Radius of the semi-circle part of the lobe trailing edge $(\mathrm{m})$

Re Reynolds number $R e=\frac{\rho \bar{U} \lambda}{\mu}=\frac{\bar{U} \lambda}{v_{\lambda}}$

St $\quad$ Strouhal number $S t=\frac{\lambda^{\mu}}{\lambda_{\mathrm{K}-\mathrm{H}}}=\frac{f \times \lambda}{\bar{U}}$

$U_{1}$ and $U_{2} \quad$ Upper and lower stream velocity $(\mathrm{m} / \mathrm{s})$

$\bar{U} \quad$ Mean velocity (nominal velocity) $\bar{U}=\frac{U_{1}+U_{2}}{2}$, $(\mathrm{m} / \mathrm{s})$

$\bar{U}, \bar{V}, \bar{W} \quad$ Mean velocities in the streamwise, spanwise and traverse directions, respectively $(\mathrm{m} / \mathrm{s})$

$u^{\prime}, v^{\prime}, w^{\prime} \quad$ rms (root-mean-square) velocities in the streamwise, spanwise and traverse directions, respectively $(\mathrm{m} / \mathrm{s})$

$X, Y, Z \quad$ Cartesian coordinate system (streamwise, spanwise and traverse directions accordingly)

\section{f}

$h$

$r$

$\Gamma_{\text {I }} \quad$ Mean streamwise circulation of the plane I,

$\varepsilon$

$\lambda \quad$ Wavelength of the lobe $(\mathrm{m})$

Kelvin-Helmholtz vortex shedding frequency $(\mathrm{Hz})$

Height of the lobe (m)

Velocity ratio of the on-coming streams, $r=\frac{U_{1}}{U_{2}}$ $\Gamma_{\mathrm{I}}=\int \frac{\mathrm{AB}}{\vec{V} \times \mathrm{d} y+\int \overrightarrow{\mathrm{BC}}} \bar{W} \times \mathrm{d} z$

$+\int_{\overrightarrow{\mathrm{CD}}} \bar{V} \times \mathrm{d} y+\int_{\overrightarrow{\mathrm{DA}}} \bar{W} \times \mathrm{d} z$

Penetration angle (half divergence angle) of the lobe-forced mixer 


$$
\begin{array}{ll}
\lambda_{K-H} & \text { Average } \mathrm{K}-\mathrm{H} \text { vortex wavelength }(\mathrm{m}) \\
\omega & \text { vorticity } \vec{\omega}=\nabla \times \vec{U} \\
\omega_{x} & \text { Streamwise vorticity } \overrightarrow{\omega_{x}}=\left(\frac{\partial W}{\partial y}-\frac{\partial V}{\partial z}\right) \times \vec{i}
\end{array}
$$

\section{Subscripts}

1 Upper stream

2 Lower stream

$\mathrm{C}$ Point $\mathrm{C}$ of the mixer trailing edge

$\mathrm{L}$ Point $\mathrm{L}$ of the mixer trailing edge

\section{Introduction}

The mixing between two co-flowing streams is an important problem in the area of fluid mechanics. Dynamic instability exists in the mixing shear layer, and it will usually lead to the generation of the spanwise vortices, namely the Kelvin-Helmholtz $(\mathrm{K}-\mathrm{H})$ vortices. Another kind of vortex, namely the streamwise vortex, may appear together with the $\mathrm{K}-\mathrm{H}$ vortices. It is a result of the energy transfer from the primary stream to the secondary stream through viscous mixing. To generate strong streamwise vortices, two control methods are usually adopted-active control and passive control. Active control is achieved by perturbing the basic flows through supplying external energy either mechanically or acoustically so as to produce certain kinds of desirable effects (Cheng and Zhou 2008). Passive flow control, on the other hand, involves no external force input. The effects of passive control are normally achieved by changing the geometries, or by adding external controllers, e.g., by placing a tripping wire to a bluff body. Compared with the active control, there is no moving part in the passive control. The lobe-forced mixer is one typical example for the passive control.

Lobe-forced mixers are splitter plates with convoluted trailing edges designed to generate large-scale streamwise vorticity between two co-flowing streams. As typical passive vortex generators, their penetration regions are three dimensionally contoured surfaces. Lobe-forced mixer has been applied to enhance mixing between two unidirectional streams efficiently, especially on the aero-fan engine exhausts as an efficient ejector. While ejectors have tremendous potential in aircraft application based on analytical predictions, the ability to implement them in effective system applications remains limited. One major reason is the slow mixing rate associated with conventional ejectors. Conventional ejectors rely on shear layer mixing only, which requires long mixing ducts. This may increase the wall friction losses, duct weight and costs. Since 1970s, many turbo-fan engines such as RB211-524G/H (UK), CFM56-5c-2 (USA), F404-GE-F1D2 (USA) have adopted the lobe-forced mixers onto their exhausts to mix the core and bypass flows more effectively within a shorter possible distance at less pressure losses (Shumpert 1980). This kind of ejectors was found to be capable of achieving either one or a combination of the following performances:

(1) Enhancing the thrust of the engines by 5\%;

(2) reducing the length of the exhausters;

(3) reducing the fuel consumption by $2.5-3.0 \%$ (Kuchar and Chamberlin 1980);

(4) reducing the noise by $3.8 \mathrm{~dB}$ (Crouch et al. 1976);

(5) reducing the infrared radiation of the stealth planes by $95 \%$.

The applications of lobe-forced mixers in the turbo-fan engine ejectors have been studied previously by many researchers. Bevilaqua (1978), Povinelli et al. (1980) and Paterson (1982) found that there were strong streamwise vortices generated in the wake of lobe-forced mixers. Experimental investigations by Skebe et al. (1988) and Barber et al. (1988), and the computational studies by O'Sullivan et al. (1996) found that both the lobe shape and its penetration angle were important parameters in determining the effectiveness of mixers. Velocity measurements by McCormick and Bennett (1994) using the triple-sensor hot film probe concluded that the intense small-scale turbulence and mixing occurred at about two to four wavelengths downstream of the mixer trailing edge was due mainly to the deformation of the $\mathrm{K}-\mathrm{H}$ vorticity into a pinched-off structure by the streamwise vorticity. The measurement of $\mathrm{Yu}$ et al. (1995) with initial turbulent boundary layers suggested that the appearance of the high turbulence region at around two to three wavelengths downstream of the trailing edge was a consequence of the positive production of Reynolds stresses; and the flow became more homogeneous at the further downstream stations. Similar conclusions were drawn by Ukeiley et al. (1993).

Previous studies concentrated on the streamwise vorticity in the wake of the lobe as it is one of the two most important factors for the enhanced mixing characteristics of the lobe-forced mixers. Another factor, the increased mixing interfacial area, is easily known directly from the trailing edge geometry. However, it is still not clear as to the individual effects of the streamwise vortices and the $\mathrm{K}-\mathrm{H}$ vortices to the mixing characteristics of the lobe-forced mixers. The present investigation will focus on how these two kinds of vortices affect the mixing characteristics, respectively. The individual aerodynamic characteristics of the $\mathrm{K}-\mathrm{H}$ vortices and the streamwise vortices would be examined and evaluated. Spectrum analysis would be adopted to examine the characteristics of the $\mathrm{K}-\mathrm{H}$ vortices while laser Doppler anemometer (LDA) measurements would be used to examine the streamwise vortices. In particular, how the geometrical configurations and modifications affect these two types of vortices and their further 
mixing performance would be studied. It should be noted that mixers with similar geometry had been investigated previously by $\mathrm{Yu}$ et al. (2000) with multiple lobes. The influence from the adjacent lobes was found to appear at a short distance (typical two wavelengths) from the trailing edge. Hence, the present investigation adopted a single lobe configuration in order to eliminate this effect.

\section{Experimental arrangements}

The experiments were carried out in an open circuit suction type wind tunnel with a test section of $183 \mathrm{~mm}$ high, $183 \mathrm{~mm}$ wide and $500 \mathrm{~mm}$ long as shown in Fig. 1. There is a flat splitter plate embedded along the centerline from the bell mouth to the contraction cone to separate the wind tunnel into two equal halves. The turbulence level of the free on-coming streams is measured to be about $1 \%$. On either side of the splitter plate, the non-uniformity levels of the free on-coming streams are less than $2 \%$, and the flow angle deviating from the axial direction of the wind tunnel is less than $0.5^{\circ}$. The temperature of the air was kept constant at $T=20^{\circ} \mathrm{C}$ during the experiments. By incorporating two different layers of screen on the upper and lower half of the bell-mouth, the velocities of the upper $\left(U_{1}\right)$ and lower $\left(U_{2}\right)$ streams can be varied from 0 to $10.4 \mathrm{~m} / \mathrm{s}$. Two velocity ratios $r\left(\equiv U_{1} / U_{2}\right)$ of 1 and 0.4 have been tested. The Reynolds number is varied from about 9,200-40,000, which was based on the mean velocity of the on-coming flows and the wavelength of the mixer models $(\lambda=60 \mathrm{~mm}$, see Fig. 2), i.e.,

$R e=\frac{\bar{U} \times \lambda}{\gamma}$,

where $\bar{U}$ is the averaged velocity of the upper and lower air flows:

$\bar{U}=\frac{U_{1}+U_{2}}{2}$.

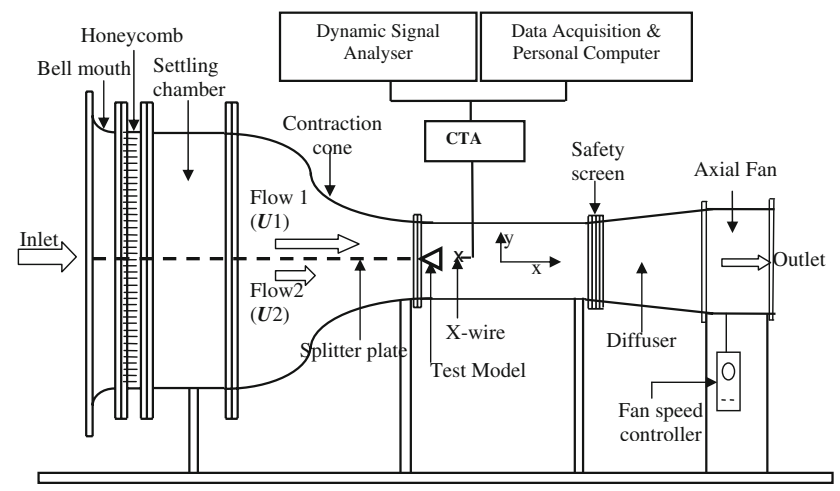

Fig. 1 Experimental arrangements
The test model was mounted at the end of the flat splitter plate located at the entrance to the test section of the wind tunnel. The flat splitter plate is made of $2 \mathrm{~mm}$ thick fiberglass. The geometries of different mixer models, which are made of $2 \mathrm{~mm}$ thick fiberglass with blunt trailing edges, are shown in Fig. 2. For the semicircular-lobe forced mixer (basic model a, shown in Fig. 2a), the wavelength and height of the trailing edge is 60 and $30 \mathrm{~mm}$, respectively, with a penetration angle of $22^{\circ}$ at the divergence section. These geometric parameters have been proven to be efficient for rapid mixing ( $\mathrm{Yu}$ and $\mathrm{Xu}$ 1998). Besides the basic mixer, mixers of different configurations, i.e., the rectangular mixer with rectangular trailing edge (model b, shown in Fig. 2b), and the triangular mixer with triangular trailing edge (model c, shown in Fig. 2c) have also been tested. Meanwhile, models with scalloping and scarfing effects were designed (models $d$ and e). The schematic diagrams of models $d$ and e are shown in Fig. $2 d$ and e, respectively, both of which were modified from the basic model a. Scalloping was achieved by eliminating up to $70 \%$ of the sidewall areas of the basic model a at the penetration region; and scarfing was to eliminate the penetration region of the basic mixer a by a half angle, i.e., $\theta=\left(90^{\circ}-\varepsilon\right) / 2=34^{\circ}$ from the side view. The Cartesian coordinate system adopted in the present investigation is shown in Fig. 3. The trailing edge of the basic mixer model consists of two different parts: a semi-circle part (denoted as part ' $C$ ') and a parallel sidewall part (denoted as ' $L$ ').

Upstream of the trailing edge, on either side of the splitter plate, there is the same area of flow. However, it should be noted that at the trailing edge, the cross-sectional area of the two sides will be changed due to the change in model geometries. The variations in the cross-sectional area at the trailing edge $(x / \lambda=0)$ are listed in Table 1 . As a result of the changes in cross-sectional areas, the velocity ratio across the splitter plate may also deviate from 1:1 to $0.4: 1$ for respective models. The averaged velocity ratios at the trailing edge are estimated and tabulated in Table 2. We shall denote this as the effects of the penetration region.

An $\mathrm{X}$-wire probe is used to measure the velocity in the wake of the mixers. The hot-wire used was Pt $10 \%-\mathrm{Rh}$ Wollaston with a diameter of $5 \mu \mathrm{m}$. The length of the wires were about $1.2-1.5 \mathrm{~mm}$. The overheat ratio was maintained at about 1.5. The signals from the hot-wire anemometers are low-pass filtered and digitized at a frequency of 2,800 Hz.

Laser Doppler anemometer has also been used to evaluate the streamwise vortices by measuring the threedimensional velocities over the region of $-0.75 \leq$ $y / \lambda \leq+0.75$ and $-1 \leq z / \lambda \leq+1$ at downstream locations $x / \lambda=0.5,1,1.5,2,3,4,5$ and 6 . At each crosssectional plane, there were about 500 measuring points. 
Fig. 2 Schematic diagrams of the test models (dimension in $\mathrm{mm}$ )

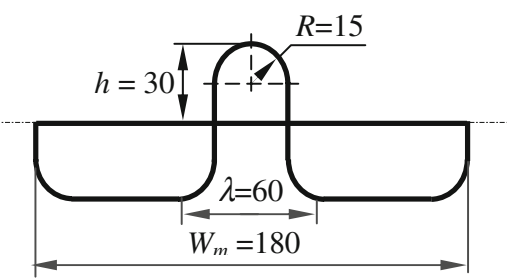

(a1) End view

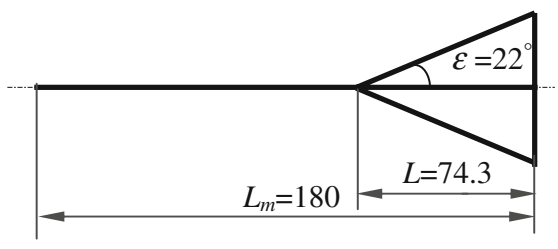

(a2) Side view (a) Semicircular-lobe forced mixer (basic model a)

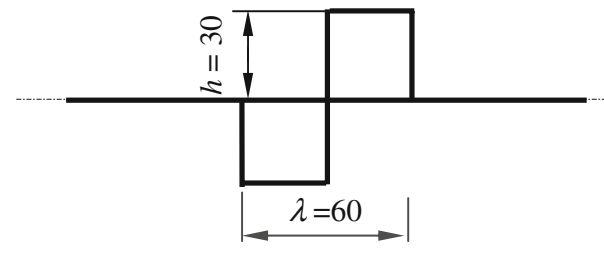

(b1) End view

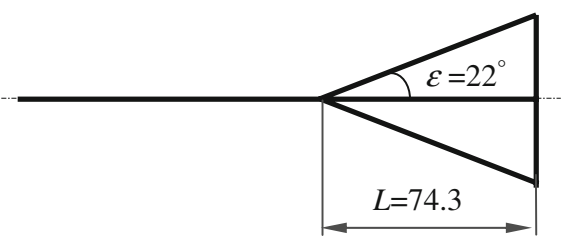

(b2) Side view (b) Rectangular-lobe forced mixer (model b)

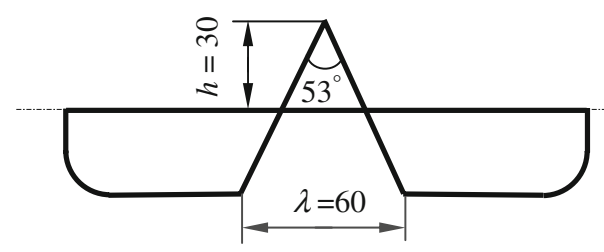

(c1) End view

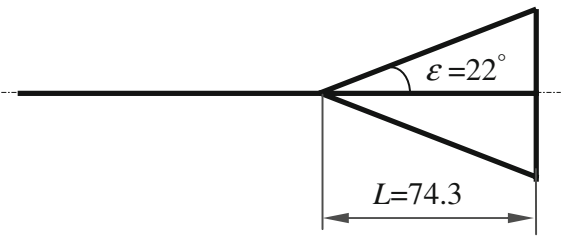

(c2) Side view (c) Triangular-lobe forced mixer (model c)

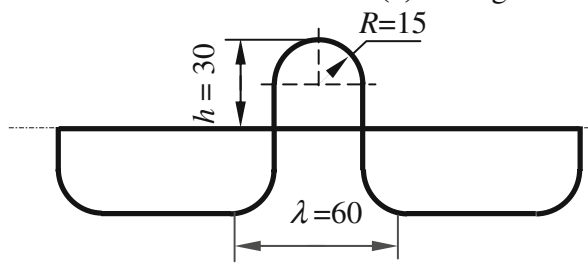

(d1) End view

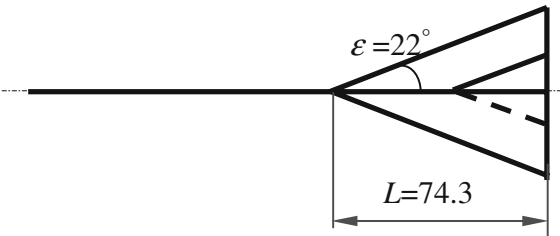

(d2) Side view (d) Scalloped-lobe forced mixer (model d)

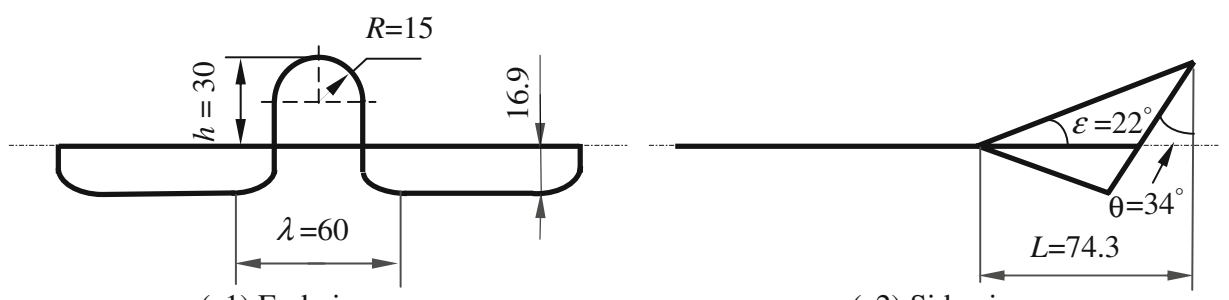

(e1) End view

(e) Scarfed-lobe forced mixer (model e)

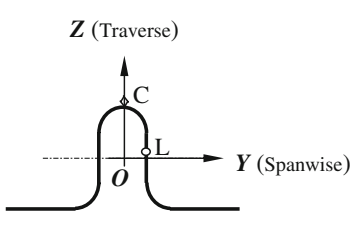

(a) End view

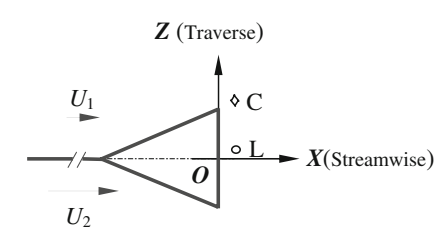

(b) Side view

Legend:

$\diamond$ Point C: (semicircle part)

- Point L: (parallel sidewall)

Fig. 3 Cartesian-coordinate system and the measuring locations $\mathrm{C}$ and $\mathrm{L}$ for the semicircular-lobe forced mixer (basic model a)

The LDA is a six-beam three-component fiber-optic laser Doppler anemometer (TSI) with a $3 \mathrm{~W}$ water-cooled argon-ion laser operating in a backward scattered mode. A focusing lens of $350 \mathrm{~mm}$ provided a measuring probe volume of $0.09 \mathrm{~mm} \times 0.09 \mathrm{~mm} \times 1.31 \mathrm{~mm}$ in the vertical direction and $0.086 \mathrm{~mm} \times 0.086 \mathrm{~mm} \times 1.24 \mathrm{~mm}$ in the horizontal direction. The fiber-optic probe was mounted on an automated three-dimensional traversing system with an accuracy of $0.01 \mathrm{~mm}$. Bragg shifting of frequency up to $40 \mathrm{MHz}$ (on each channel) was used to avoid directional ambiguity. The Doppler signals were detected by 
Table 1 Percentages of the upper and lower side areas of different test models

\begin{tabular}{llllll}
\hline $\begin{array}{l}\text { Models percentage } \\
\text { of area (\%) }\end{array}$ & a (basic) & b & c & d & e \\
\hline Upper side & 59 & 50 & 59 & 59 & 55 \\
Lower side & 41 & 50 & 41 & 41 & 45 \\
\hline
\end{tabular}

Table 2 Averaged velocity ratio at the trailing edge $(x / \lambda=0)$ for different test models

\begin{tabular}{llllll}
\hline $\begin{array}{l}\text { Models } \\
\text { velocity ratio } r\end{array}$ & a (basic) & b & $c$ & $\mathrm{~d}$ & $\mathrm{e}$ \\
\hline $\begin{array}{c}\text { Nominal velocity } \\
\text { ratio 1:1 }\end{array}$ & $0.69: 1$ & $1: 1$ & $0.69: 1$ & $0.69: 1$ & $0.82: 1$ \\
$\begin{array}{l}\text { Nominal velocity } \\
\text { ratio 0.4:1 }\end{array}$ & $0.28: 1$ & $0.4: 1$ & $0.28: 1$ & $0.28: 1$ & $0.33: 1$ \\
\hline
\end{tabular}

photo-multipliers and processed by burst spectrum analyzers (IFA 750). Fine water particles with a diameter of 5-10 $\mu \mathrm{m}$ generated by a commercial nebulizer were used to seed the airflow. Except in some regions immediately behind the trailing edge, data rates of $500-1,000 \mathrm{~Hz}$ were normally obtainable. At each measuring point, the mean velocities together with the corresponding fluctuations (rms) were determined from more than 4,000 samples together with a coincidence window of $1 \mu \mathrm{s}$. Other coincidence window widths from 1 to $100 \mu$ s were also tested but no significant differences in the results were found. Considering the satisfactory data rates, the samples were reasonably assumed to be normally distributed. The sources of error were mainly from velocity biasing, velocity gradient broadening (Durst et al. 1981), the accuracy of the signal processor and the finite sampling size (Yanta and Smith 1973). In the present study, these four items were found to be about 1.5, 1, 0.8 and $0.04 \%$, respectively. By using the method of propagation of errors (Moffat 1988), the uncertainty of the measured mean-velocity component is estimated to be about 2\%. Details of the uncertainty analysis can be found in Mao (2005).

\section{Results and discussion}

\section{$3.1 \mathrm{~K}-\mathrm{H}$ vortices}

\subsubsection{Semicircular-lobe forced mixer (basic mixer a)}

The vortex shedding frequency of the $\mathrm{K}-\mathrm{H}$ vortices can be recognized at the frequency where the energy spectrum showed a clear peak. As measured in the experiments, there were always two main frequencies in the wake of the basic mixer a due to the fact that the initial conditions at points $\mathrm{C}$ and $\mathrm{L}$ were different significantly. In the wake region of the semicircle part of the trailing edge, as shown in Fig. 3, the dominant frequency was essentially a constant value denoted as $f_{\mathrm{C}}$; while for the regions in the wake of the parallel sidewalls of the trailing edge, the dominant frequency was also a constant value denoted as $f_{\mathrm{L}}$. Figure 4 shows two typical spectra of the basic mixer for the velocity condition $U_{1}=U_{2}=5.66 \mathrm{~m} / \mathrm{s}$ at points $\mathrm{C}$ and $\mathrm{L}$, respectively. In the figure the frequency was normalized using $\lambda$ and $\bar{U}$. With this normalization, the peak frequency corresponded to the Strouhal number, as defined later. It can be seen that the $\mathrm{K}-\mathrm{H}$ vortices were shed at $f_{\mathrm{C}}=560 \mathrm{~Hz}$ or a non-dimensional frequency of 5.93 at point $\mathrm{C}$. At point $\mathrm{L}$, the $\mathrm{K}-\mathrm{H}$ vortices were shed at $f_{\mathrm{L}}=480 \mathrm{~Hz}$ or a non-dimensional frequency of 5.09. There are harmonics on the two spectra. The peak in Fig. 4a is more significant than that in Fig. 4b. This result may indicate that the vortices generated in the former is much stronger than that in the latter. Figure 5 shows the frequency variations of the basic mixer at all velocities involved in the present investigation. It is very clear that $f_{\mathrm{C}}$
Fig. 4 Typical power spectra of the semicircular-lobe forced mixer (basic model a)

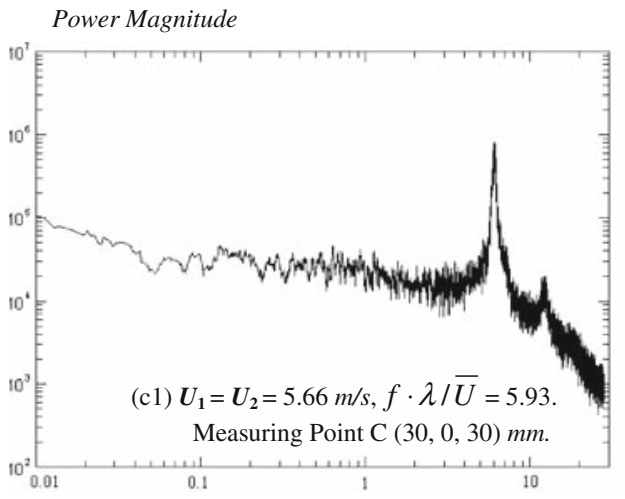

(a) Point $\mathrm{C} \quad f \cdot \lambda / \bar{U}$

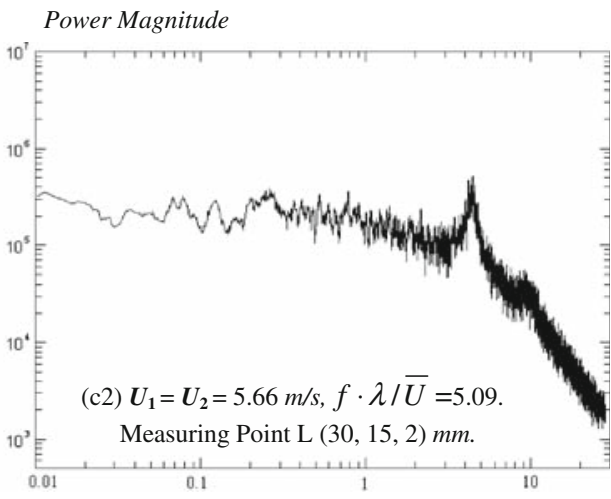

(b) Point L 


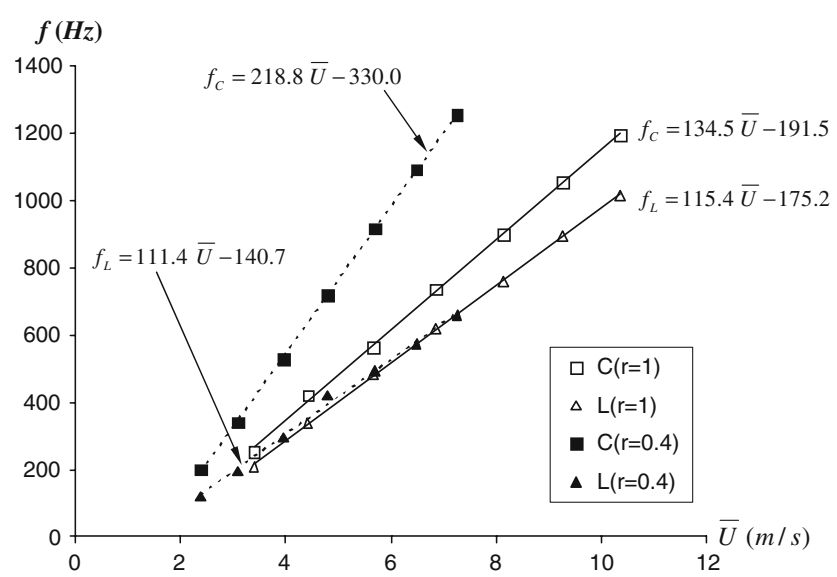

Fig. $5 \mathrm{~K}-\mathrm{H}$ vortex shedding frequencies after the trailing edge of the semicircular-lobe forced mixer (basic model a)

was always higher than $f_{\mathrm{L}}$ under the same on-coming velocity condition. The velocity ratio can be much more affective to the frequency at point $\mathrm{C}$ than that at point $\mathrm{L}$. For the basic mixer a, when the velocity ratio decreases from 1 to 0.4 the frequency at point $\mathrm{C}$ increased by $100 \%$ at the same mean velocity. However, the velocity ratio had little effects at point $\mathrm{L}$ on its $\mathrm{K}-\mathrm{H}$ vortex shedding frequency. By applying linear regressions to the measured data, the correlations between the vortex shedding frequency and the mean on-coming velocity may be approximated as:

$$
\begin{aligned}
f_{\mathrm{C}}= & 134.5 \bar{U}-191.5 \\
& (r=1,3.40 \mathrm{~m} / \mathrm{s} \leq \bar{U} \leq 10.36 \mathrm{~m} / \mathrm{s}) ; \\
f_{\mathrm{L}}= & 115.4 \bar{U}-175.2 \\
& (r=1,3.40 \mathrm{~m} / \mathrm{s} \leq \bar{U} \leq 10.36 \mathrm{~m} / \mathrm{s}) ; \\
f_{\mathrm{C}}= & 218.8 \bar{U}-330.0 \\
& (r=0.4,2.39 \mathrm{~m} / \mathrm{s} \leq \bar{U} \leq 7.25 \mathrm{~m} / \mathrm{s}) ; \\
f_{\mathrm{L}}= & 111.4 \bar{U}-140.7 \\
& (r=0.4,2.39 \mathrm{~m} / \mathrm{s} \leq \bar{U} \leq 7.25 \mathrm{~m} / \mathrm{s}) .
\end{aligned}
$$

To examine periodic wakes, the Roshko number was usually adopted (Roshko 1955). The frequency can be correlated to the Roshko number based on the wavelength $\lambda$ of the lobe and the kinematic viscosity $v$ of the air, i.e.,

$R o=\frac{f \times \lambda^{2}}{v}$.

From the relationship between the $\mathrm{K}-\mathrm{H}$ vortex shedding frequency and the mean velocity, it is easy to deduce that the Roshko number increases linearly with the increase of the Reynolds number at both points $\mathrm{C}$ and $\mathrm{L}$. The following relations at points $\mathrm{C}$ and $\mathrm{L}$ for the two different velocity ratios could be obtained from the aforementioned Eqs. 1-7:

$$
\begin{aligned}
R o_{\mathrm{C}}= & 8.07 R e-44,300 \\
& (r=1,13,100 \leq R e \leq 40,000) ; \\
R o_{\mathrm{L}}= & 6.92 R e-40,500 \\
& (r=1, \quad 13,100 \leq R e \leq 40,000) ; \\
R o_{\mathrm{C}}= & 13.1 R e-76,300 \\
& (r=0.4, \quad 9,200 \leq R e \leq 27,900) ; \\
R o_{\mathrm{L}}= & 6.68 R e-32,500 \\
& (r=0.4, \quad 9,200 \leq R e \leq 27,900) .
\end{aligned}
$$

Although the increases of the Roshko number with Reynolds number are linear at points $\mathrm{C}$ and $\mathrm{L}$ for $r=1$ and 0.4 , their magnitudes of the increasing rates are different.

The Strouhal number represents the increasing rate of the Roshko number with Reynolds number,

$S t=\frac{R o}{R e}$.

Actually it is also the ratio between the nominal length $(\lambda)$ and the mean wavelength of the $\mathrm{K}-\mathrm{H}$ vortices:

$S t=\frac{f \times \lambda}{\bar{U}}=\frac{\lambda}{\lambda_{\mathrm{K}-\mathrm{H}}}$.

Therefore, from Eq. 13, it is clear that the physical meaning of the Strouhal number is how many roll-ups are contained within each nominal length (lobe wavelength $\lambda=60 \mathrm{~mm}$ here) in the streamwise direction.

According to the relations of the Roshko number, i.e., Eqs. 8-11, the Strouhal number for the basic mixer (model a) at points $\mathrm{C}$ and $\mathrm{L}$ for the two different velocity ratios can be obtained as follows:

$S t_{\mathrm{C}}=8.07-\frac{44,300}{\operatorname{Re}} \quad(r=1, \quad 13,100 \leq \operatorname{Re} \leq 40,000) ;$

$S t_{\mathrm{L}}=6.92-\frac{40,500}{R e} \quad(r=1, \quad 13,100 \leq \operatorname{Re} \leq 40,000) ;$

$S t_{\mathrm{C}}=13.1-\frac{76,300}{\operatorname{Re}} \quad(r=0.4, \quad 9,200 \leq R e \leq 27,900) ;$

$S t_{\mathrm{L}}=6.68-\frac{32,500}{R e} \quad(r=0.4, \quad 9,200 \leq R e \leq 27,900)$.

These results are shown in Fig. 6. It can be easily deduced that Strouhal number increases with increasing Reynolds number while the increasing rate decreases:

Rate of increase $=\frac{\mathrm{d}(S t)}{\mathrm{d}(R e)} \propto \frac{1}{(R e)^{2}}$.

The Strouhal number increases rapidly when Reynolds number changes from 9,200 to about 20,000. When 


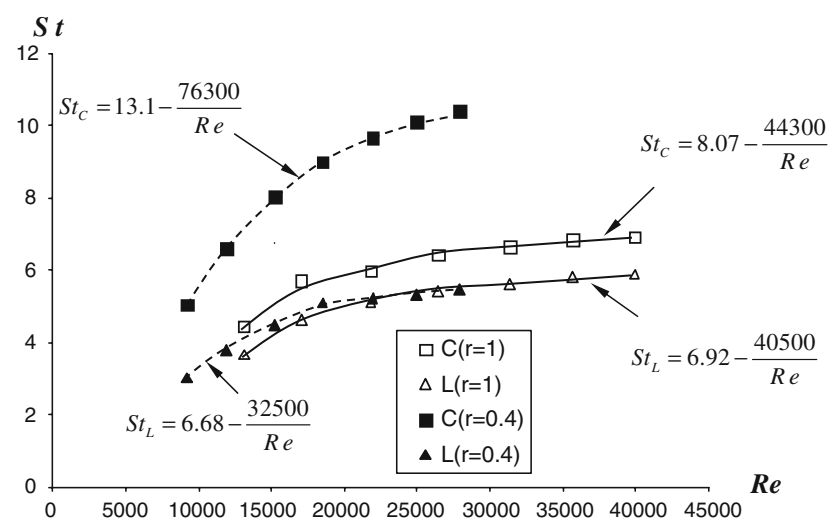

Fig. 6 Strouhal number variation with Reynolds number for the semicircular-lobe forced mixer (basic model a)

Reynolds number is larger than 20,000, the Strouhal number increases relatively slowly. As a result, constant maximum Strouhal number values are expected to be approached at very high Reynolds number. This is because the viscous force and the inertial force play different roles corresponding to different Reynolds number ranges. From Eq. 13 it can be seen that the higher the Strouhal number, the smaller the wavelength of the $\mathrm{K}-\mathrm{H}$ vortex. The lobeforced mixer is therefore expected to achieve better mixing at higher Strouhal number. The relationship between the Strouhal number and Reynolds number provides some interesting information. From the viewpoint of the $\mathrm{K}-\mathrm{H}$ vortices, when Reynolds number is low, the mixing performance of the forced mixer increases with Reynolds number; but once Reynolds number is very high the mixing performance contributed from the $\mathrm{K}-\mathrm{H}$ vortices approaches its maximum and becomes stable thereafter. The velocity ratio affects Strouhal number at point $\mathrm{C}$ more significantly than that at point $\mathrm{L}$.

\subsubsection{Other configurations}

Compared to the basic semicircular-lobe mixer a, the K-H vortex shedding frequency, the Roshko and Strouhal numbers for the other four models all revealed the same trends as the basic mixer a. So only some results from the other models are shown as follows here. The vortex shedding frequency and Strouhal number for the rectangular model b, for example, have been shown in Figs. 7 and 8, respectively. Figure 9 is the Strouhal number variations for the scalloped-lobe mixer $\mathrm{d}$ and scarfed-lobe mixer e at point $\mathrm{C}$. It is rather clear that the linear variation tendency for the $\mathrm{K}-\mathrm{H}$ vortex shedding frequency and the nonlinear variation tendency for the Strouhal number were both the same as the basic model a, although their rates of increase were different.

For all the tested models, their maximum Strouhal numbers have been listed in Table 3. From the table it is

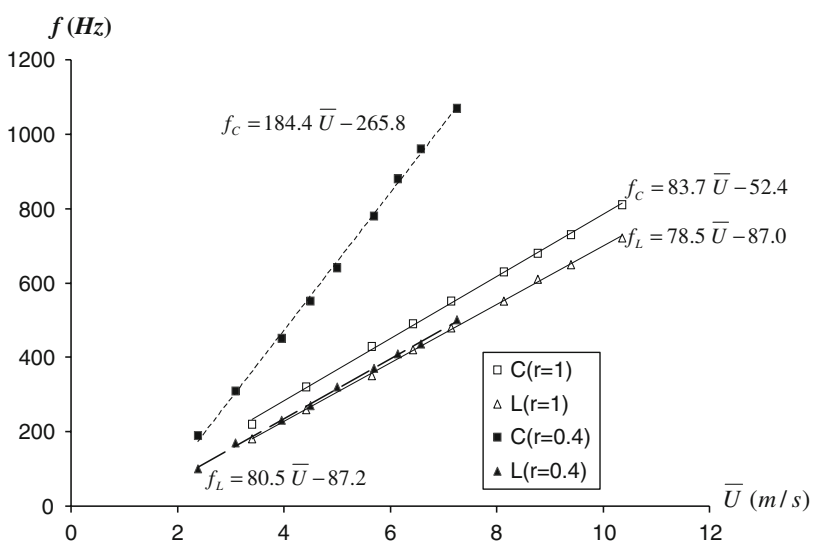

Fig. $7 \mathrm{~K}-\mathrm{H}$ vortex shedding frequencies after the trailing edge of the rectangular-lobe forced mixer (model b)

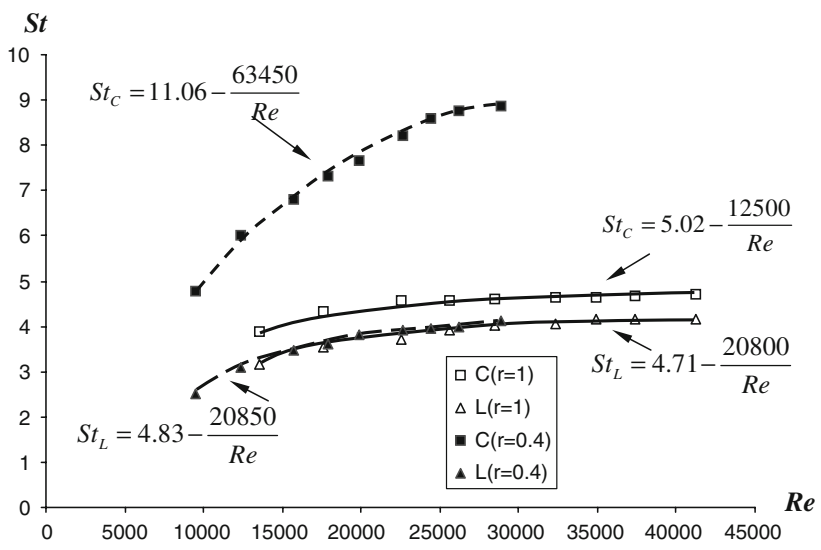

Fig. 8 Strouhal number variation with Reynolds number for the rectangular-lobe forced mixer (model b)

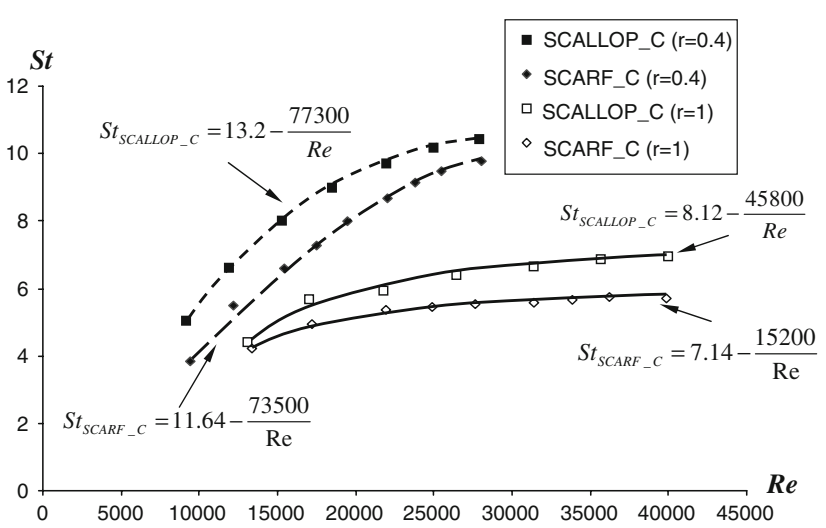

Fig. 9 Strouhal number variation with Reynolds number for the scalloped-lobe forced mixer (model d) and scarfed-lobe forced mixer (model e) at Point C

clear that for models $\mathrm{b}$ and $\mathrm{c}$, their maximum Strouhal numbers are smaller than the basic semicircular model a. The latter has smaller $\mathrm{K}-\mathrm{H}$ mean wavelength than the 
Table 3 Maximum Strouhal numbers for different test models

\begin{tabular}{lllll}
\hline Points models & $\mathrm{C}(r=1)$ & $\mathrm{L}(r=1)$ & $\mathrm{C}(r=0.4)$ & $\mathrm{L}(r=0.4)$ \\
\hline Basic model a & 8.07 & 6.92 & 13.1 & 6.68 \\
Rectangular model b & 5.02 & 4.71 & 11.06 & 4.83 \\
Triangular model c & - & 5.95 & - & 6.50 \\
Scalloped model d & 8.12 & - & 13.2 & - \\
Scarfed model e & 7.14 & 6.87 & 11.64 & 6.65
\end{tabular}

The dashed lines represent that point $\mathrm{C}$ or $\mathrm{L}$ does not exist in these models

former two. Furthermore, there is no point $\mathrm{C}$ for the triangular mixer (model c). As it has been shown in Fig. 4, the point $\mathrm{C}$ can usually shed stronger $\mathrm{K}-\mathrm{H}$ vortices than point $\mathrm{L}$. So the normal $\mathrm{K}-\mathrm{H}$ vortices shed after the trailing edge of the triangular mixer are extremely weak.

\subsection{Streamwise vortices}

Three-dimensional mean velocity fields and their corresponding fluctuations in the near wake of the two-stream mixing flow have been measured using LDA. The streamwise velocity, the mean velocity vectors of the secondary flow and the streamwise vorticity have been non-dimensionalized and presented as the following. The contours of the streamwise vorticity were constructed from grids at a spatial resolution of $5 \mathrm{~mm} \times 5 \mathrm{~mm}$ on the $\mathrm{y}-\mathrm{z}$ plane by the velocity components via a laser Doppler anemometer. The circulation evaluated only represents the spatially averaged strength of the streamwise vorticity for a particular plane of interest.

\subsubsection{Streamwise velocity, secondary velocity vectors and vorticity contours}

Streamwise velocity has been non-dimensionalized by the nominal velocity, or mean velocity of the two streams. Due to the symmetry of the models and the flow fields, only half lobe (the left side here) will be shown in this paper. The mean secondary velocity vector is:

$\overrightarrow{V_{\mathrm{s}}}=\bar{V} \times \vec{j}+\bar{W} \times \vec{k}$,

in which $V$ and $W$ are both non-dimensionalized by the nominal velocity. Similarly only the right half of the lobe will be shown here. The arrow base of the secondary velocity vector is at the measuring point.

3.2.1.1 Model a at velocity ratio $r=1$ Figure $10 \mathrm{~b}$ shows the velocity contours and the secondary velocity vector. For the flow field of model a, although the velocities of the upper and lower on-coming air-flows $\left(U_{1}, U_{2}\right)$ are the same, the streamwise velocity fields after the trailing edge deviate from the mean value by up to $30 \%$ due to the effects of the penetration region [Fig. 10b(1)]. At $x$ / $\lambda=0.5$, low momentum fluids could be found near the upper trough regions, while the fluids near the lower trough region accelerated. It should be noted that this difference was the direct result from the mixer geometry with only one lobe. The upper side airflow went through a divergent passage, while the lower side airflow went through a convergent one. No flow separations were detected at both troughs. The asymmetrical geometry of the mixer led to the asymmetrical flow fields. This also led to the streamwise vorticity asymmetry-the streamwise vortices generated here were different from those generated after the multilobe forced mixer, as typically measured by Eckerle et al. (1992). The maximum value of the secondary flow was about $60 \%$ of the mean streamwise velocity at this station. Figure 11a shows the streamwise velocity profiles at $y / \lambda=3 / 4,0$ and $-3 / 4$, respectively. The symmetry of the velocity profiles at $y / \lambda=3 / 4$ and $-3 / 4$ is very good. The two troughs of velocity profiles indicated that low momentum fluids were accumulated near the upper trough of the lobe shown in Fig. 10a. At $y=0$, the velocity profile showed small fluctuations because both the velocity magnitude and its direction were altered especially near the upper peak and lower trough of the lobe.

At $x / \lambda=1$ [Fig. 10b(2)], the streamwise velocity contours began to diffuse. The secondary velocity vectors were the strongest at this station with a maximum value of about $0.75 \bar{U}$. At $x / \lambda=2$, the streamwise velocity contours continued to diffuse with the velocity gradients diminished gradually, as shown in Fig. 10b(3). The magnitude of the secondary flow also decreased, with a maximum values of about $0.5 \bar{U}$. At station $x / \lambda=4$ [Fig. 10b(4)], the streamwise velocity gradients reduced further. The maximum secondary flow was about $0.20-0.25 \bar{U}$ only.

The streamwise vorticity $\left(\omega_{x}\right)$ has the vector form of

$\overrightarrow{\omega_{x}}=\left(\frac{\partial W}{\partial y}-\frac{\partial V}{\partial z}\right) \times \vec{i}$

The vorticity was normalized by $(\bar{U} / \lambda)$. The contours of the vorticity at four different downstream stations $x / \lambda=0.5,1,2$ and 4 are plotted in Fig. 12a. Compared with the measured cross-section area, the streamwise vortices were localized within the areas at the same scale as the lobe wavelength. It is rather clear that the two streamwise vortices were counter-rotating, either in $+x$ or $-x$ direction. They were formed immediately after the trailing edge of the mixer and were strengthened between the stations $x / \lambda=0.5$ and 1 with peak values of about \pm 1.5 ; and then dissipate gradually.

3.2.1.2 Model a at velocity ratio $r=0.4$ For this case, the velocity of the upper side on-coming airflow was $41 \%$ of the lower side. At $x / \lambda=0.5$ [Fig. 10c(1)], the streamwise 


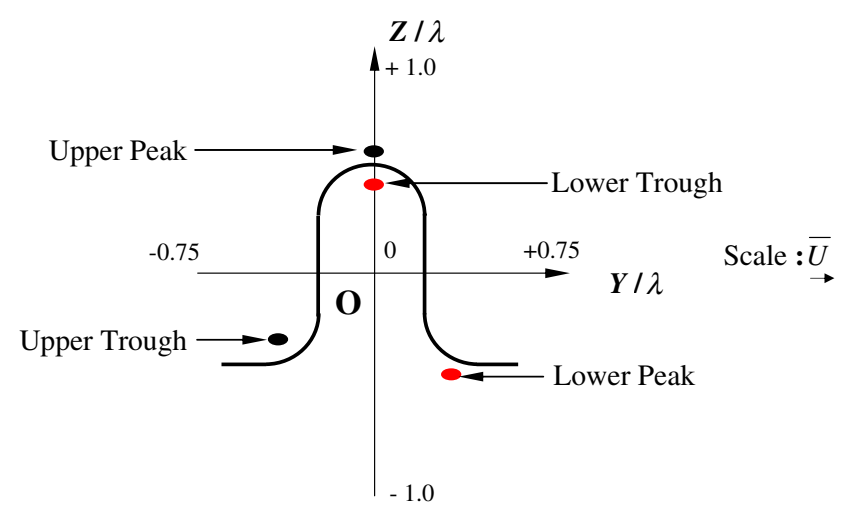

(a) Coordinate system and vector magnitude scale

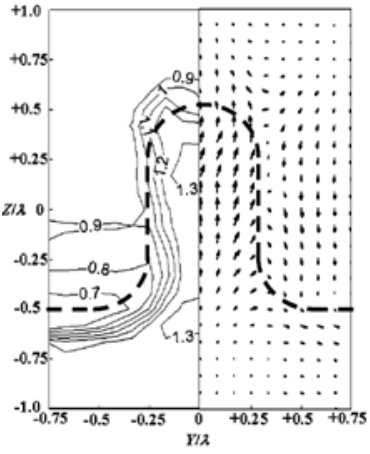

(1) $x / \lambda=0.5$

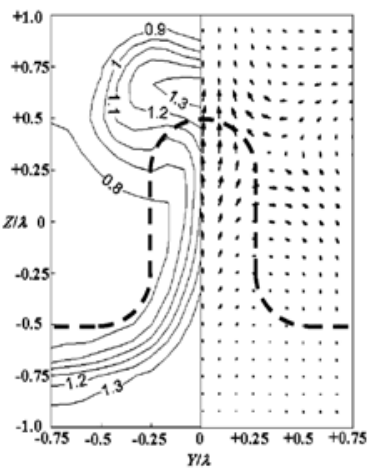

(3) $x / \lambda=2$

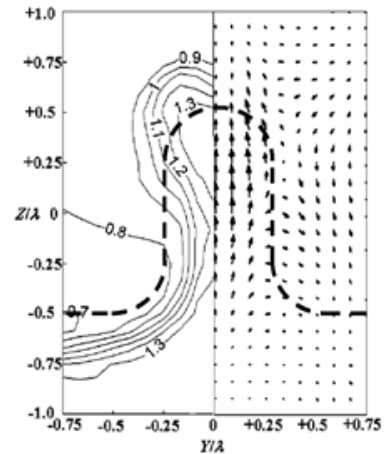

(2) $x / \lambda=1$

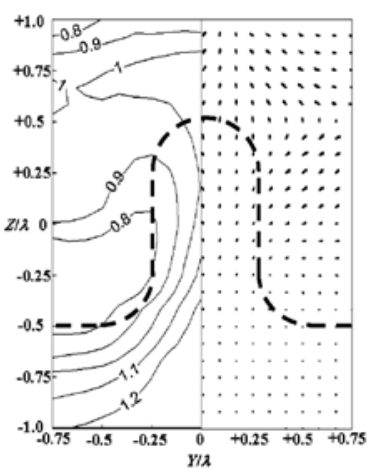

(4) $x / \lambda=4$

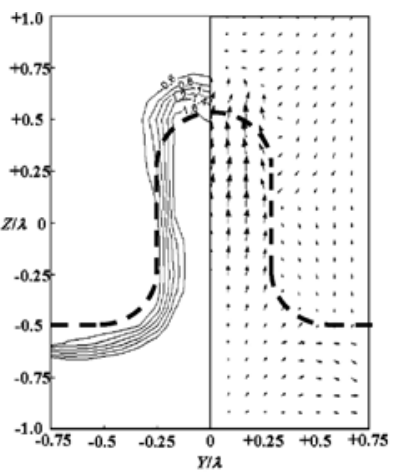

(1) $x / \lambda=0.5$

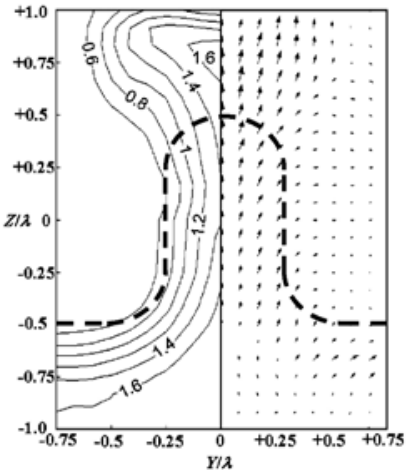

(3) $x / \lambda=2$

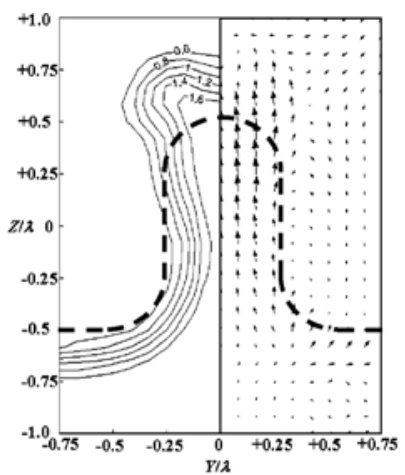

(2) $x / \lambda=1$

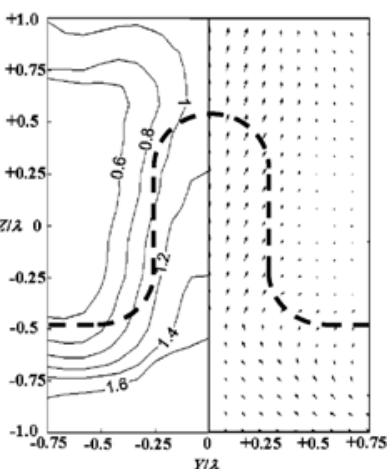

(4) $x / \lambda=4$

(b) Velocity ratio $r=1$

(c) Velocity ratio $r=0.4$

Fig. 10 Coordinate system and contours of $(U / \bar{U})$ and the secondary flow velocity vectors $\left(\overrightarrow{V_{s}} / \bar{U}\right)$ at different downstream stations after the trailing edge of the semicircular-lobe forced mixer for $r=1$ and 0.4

velocity contours were clustered near the trailing edge, and the velocity gradients there were much higher than those for the case of velocity ratio $r=1$ (see Fig. 10b). The large mean velocity gradient, or the mean shear, may indicate a better mixing effect. The large mean velocity gradient or the mean shear may indicate a better mixing effect. This explains why the $\mathrm{K}-\mathrm{H}$ vortices for this case were much stronger than the former case with the velocity of the two on- coming streams matched. The maximum value of the secondary flow was about $0.7 \bar{U}$. Additional velocity profiles at locations of $y / \lambda=3 / 4,0$ and $-3 / 4$ were shown in Fig. 11b. The velocity profile at both $y / \lambda=3 / 4$ and $-3 / 4$ experienced dramatic changes near the upper trough and lower peak of the lobe at around $z / \lambda=-0.6$. At $y=0$, however, the velocity transition was found from $z / \lambda=0.4$ to $z / \lambda=0.75$ at relatively lower velocity gradients. 
Fig. 11 Streamwise velocity profile plots $(U / \bar{U})$ at $x=30 \mathrm{~mm}$ of the basic mixer a for a velocity ratio $r=1$ and b velocity ratio 0.4

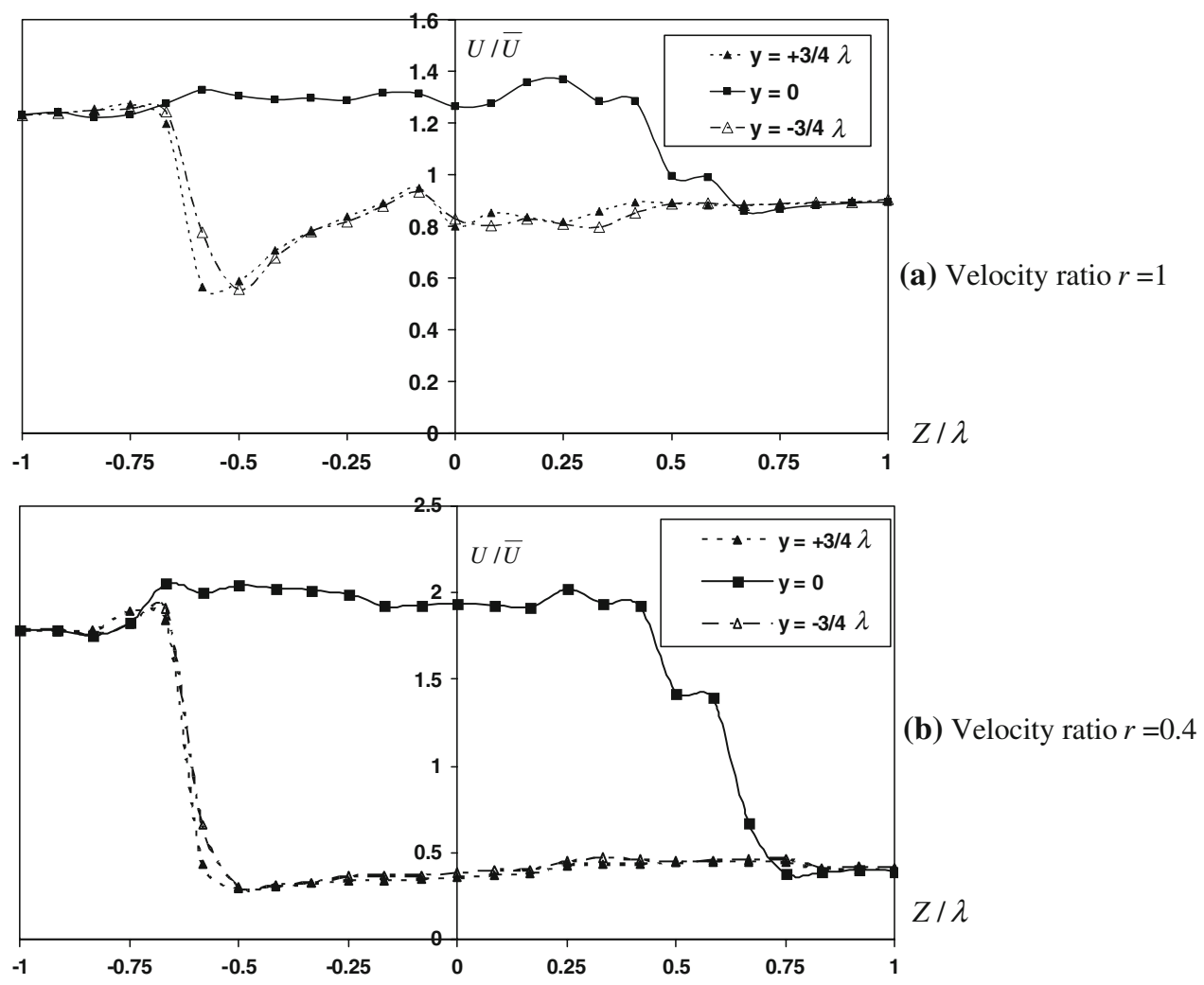

At $x / \lambda=1$, the streamwise velocity contours began to diffuse. The secondary velocity vectors peaked at this station with a maximum value of about $0.8 \bar{U}$, as shown in Fig. 10c(2). Rapid exchange of momentum between these two streams happened here, and this contributed much to the mixing enhancement. At $x / \lambda=2$ and 4 [Figs. 10c(3), (4)], the streamwise velocity contours continued to diffuse with the velocity gradients being decreased. The magnitudes of the secondary flows kept decreasing with the maximum values of about 0.55 and $0.20 \bar{U}$ at $x / \lambda=2$ and 4 , respectively. The mixing was yet to complete, and the momentum exchange between these two streams would continue downstream. The normalized streamwise vorticity contours at this velocity ratio 0.4 were plotted in Fig. $12 \mathrm{~b}$. Initially the streamwise vortices were distributed both near the lobe peak and trough at $x / \lambda=0.5$. Within the following downstream stations, however, the streamwise vortices seemed to move upwards gradually. The normalized peak vorticity for this velocity ratio were found to be around \pm 1.0 . Compared with the case at velocity ratio 1 , the streamwise vorticity for this case were weaker; and it took longer time for them to be formed and strengthened.

3.2.1.3 Other models Typical results such as the rectangular-lobe forced mixer at $r=1$ and the scalloped-lobe forced mixer at $r=0.4$ have been plotted in Figs. 13 and 14, respectively. It can be observed from Fig. 13 that the streamwise velocity of the on-coming flow decreases at both troughs up to $20 \%$ of the mean velocity. The velocity contours diffused gradually with downstream distance. By the station at $x / \lambda=4$, the velocity distribution had become almost uniform in the streamwise direction. The secondary flows were mainly restricted within the wake of the troughs. The strongest secondary flow at the first station examined was about $70 \%$ of the mean streamwise velocity scale, as shown in Fig. 13(1). It then became smaller and smaller at further downstream stations. The normalized streamwise vorticity contours have also been shown in the figure on the whole plane scheme. The peak vorticity at $x / \lambda=0.5$ were found to be about +1.5 and -1.0 . The vortex oriented in the $x$ direction located at the middle of the plane could be recognized obviously. There existed two similar vortices on the left and right sides of $y$ axis, both oriented in $-x$ direction. The vortices decayed at further downstream stations with peak vorticity being about \pm 0.8 and \pm 0.6 at $x / \lambda=2$ and 4 , respectively.

For the scalloped-lobe forced mixer at $r=0.4$, the maximum secondary velocities were about 70,62 and $35 \%$ at stations $x / \lambda=0.5,2.0$ and 4.0, respectively as shown in Fig. 14. Actually these results were very similar to the basic model a. However, small differences between these two models were that the initial secondary velocity vectors for the scalloped model were slightly stronger than those 


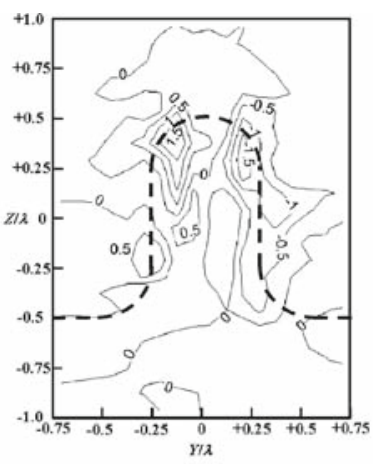

(1) $x / \lambda=0.5$

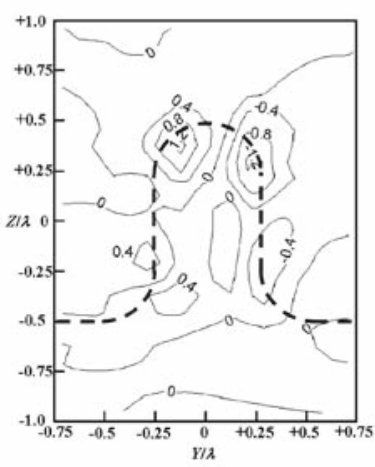

(3) $x / \lambda=2$

(a) Velocity ratio $r=1$

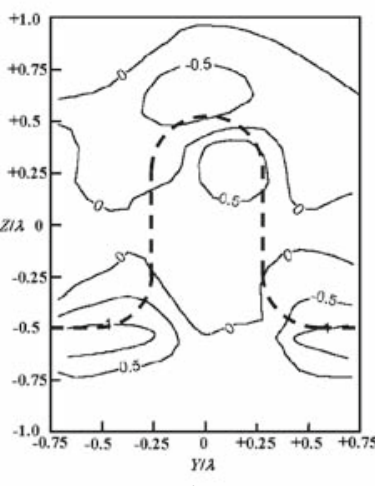

(1) $\mathrm{x} / \lambda=0.5$

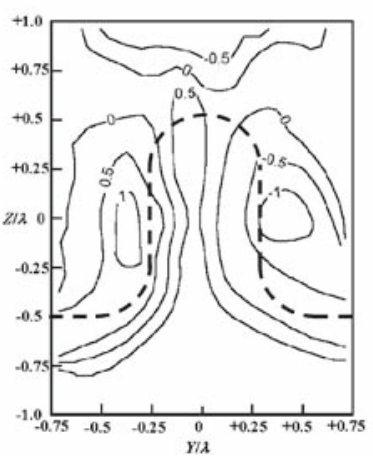

(3) $x / \lambda=2$

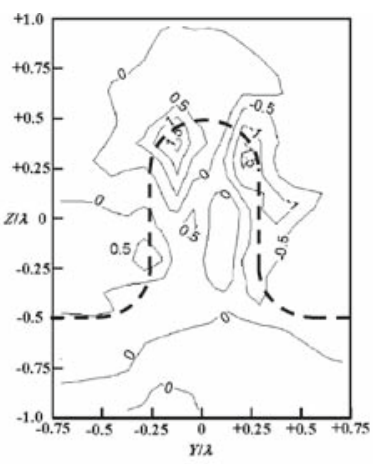

(2) $x / \lambda=1$

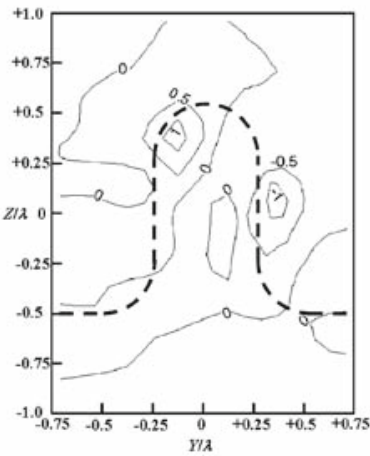

(4) $x / \lambda=4$

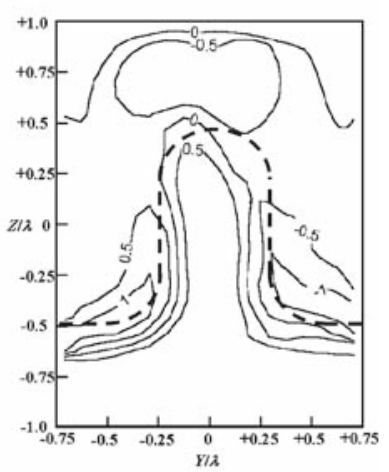

(2) $x / \lambda=1$

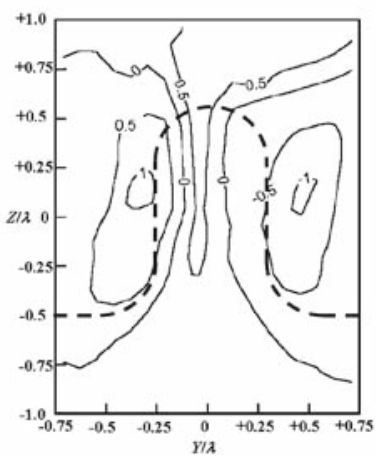

(4) $x / \lambda=4$

(b) Velocity ratio $r=0.4$

Fig. 12 Streamwise vorticity contours of the basic mixer a for a velocity ratio $r=1$ and $\mathbf{b}$ velocity ratio 0.4 for the basic model a, and the former case decayed slightly faster. These differences can be explained as follows. Owing to the scalloping effect, some additional streamwise vortices would be generated near the notches, as sketched in Fig. 15. They were rotating in the same directions as the main streamwise vortices. As a result, the secondary velocity vectors together with the total streamwise vorticity would be slightly stronger than those of basic model a. However, the consequent merging process between the additional and the main streamwise vortices would weaken the total streamwise vorticity. As far as we know, the merger with like signed strain is less efficient due to the final deformation and filamentation of the resulting vortex (Waugh 1992). The whole-plane scheme of the normalized streamwise vorticity have been calculated and shown in Fig. 14 as well. The peak vorticity at $x / \lambda=0.5$ was found to be \pm 1.6 , which was slightly higher than the basic model a of about \pm 1.5 .

\subsubsection{Mean streamwise vorticity (circulation)}

The strength of the mean streamwise vorticity $\left(\overline{\omega_{x}}\right)$, which may be considered as an important parameter to quantify the effectiveness of mixer performance, could be evaluated using the following expression:

$C_{\mathrm{I}}=\frac{\Gamma_{\mathrm{I}}}{\bar{U} \times \lambda}$,

in which $\Gamma_{\mathrm{I}}$ is the streamwise circulation of the plane $\mathrm{I}$ as shown in Fig. 16, where $\partial \mathrm{I}$ encompasses the plane I or $A(x, 0,30)-B(x, 30,30)-C(x, 30,-30)-D(x, 0,-30)$ $-A(x, 0,30)$,

$$
\begin{aligned}
\Gamma_{\mathrm{I}}= & \iint_{\mathrm{I}} \omega_{x} \times \mathrm{d} A=\overline{\omega_{x}} \times A=\oint_{\mathrm{\partial I}} \overrightarrow{V_{\mathrm{s}}} \times \mathrm{d} \vec{l} \\
= & \int_{\overrightarrow{\mathrm{AB}}} \bar{V} \times \mathrm{d} y+\int_{\overrightarrow{\mathrm{BC}}} \bar{W} \times \mathrm{d} z+\int_{\overrightarrow{\mathrm{CD}}} \bar{V} \times \mathrm{d} y \\
& +\int_{\overrightarrow{\mathrm{DA}}} \bar{W} \times \mathrm{d} z .
\end{aligned}
$$

Figure 16 shows the variations of the normalized streamwise circulation with downstream distance from the various models, including the mixers $\mathrm{a}, \mathrm{b}$ and $\mathrm{c}$, for both velocity ratios $r=1$ and 0.4 . From this figure it could be observed that the rectangular model $\mathrm{b}$ had the highest mean streamwise vorticity, followed by the basic semicircular model a. The mean streamwise vorticity magnitude in the wake of the triangular model $\mathrm{c}$ was the lowest among the three models. One reason was that there was no parallel sidewall for the triangular model, which led to serious boundary blockage effects. The circulation of the triangular model was about $60 \%$ of the basic semicircular model for both the two velocity ratios involved. 
Fig. 13 Contours of $(U / \bar{U})$, secondary flow velocity vectors $\left(\overrightarrow{V_{s}} / \bar{U}\right)$ and streamwise vorticity contours at different downstream stations after the trailing edge of rectangular-lobe forced mixer (model $b$ ) for velocity ratio $r=1$
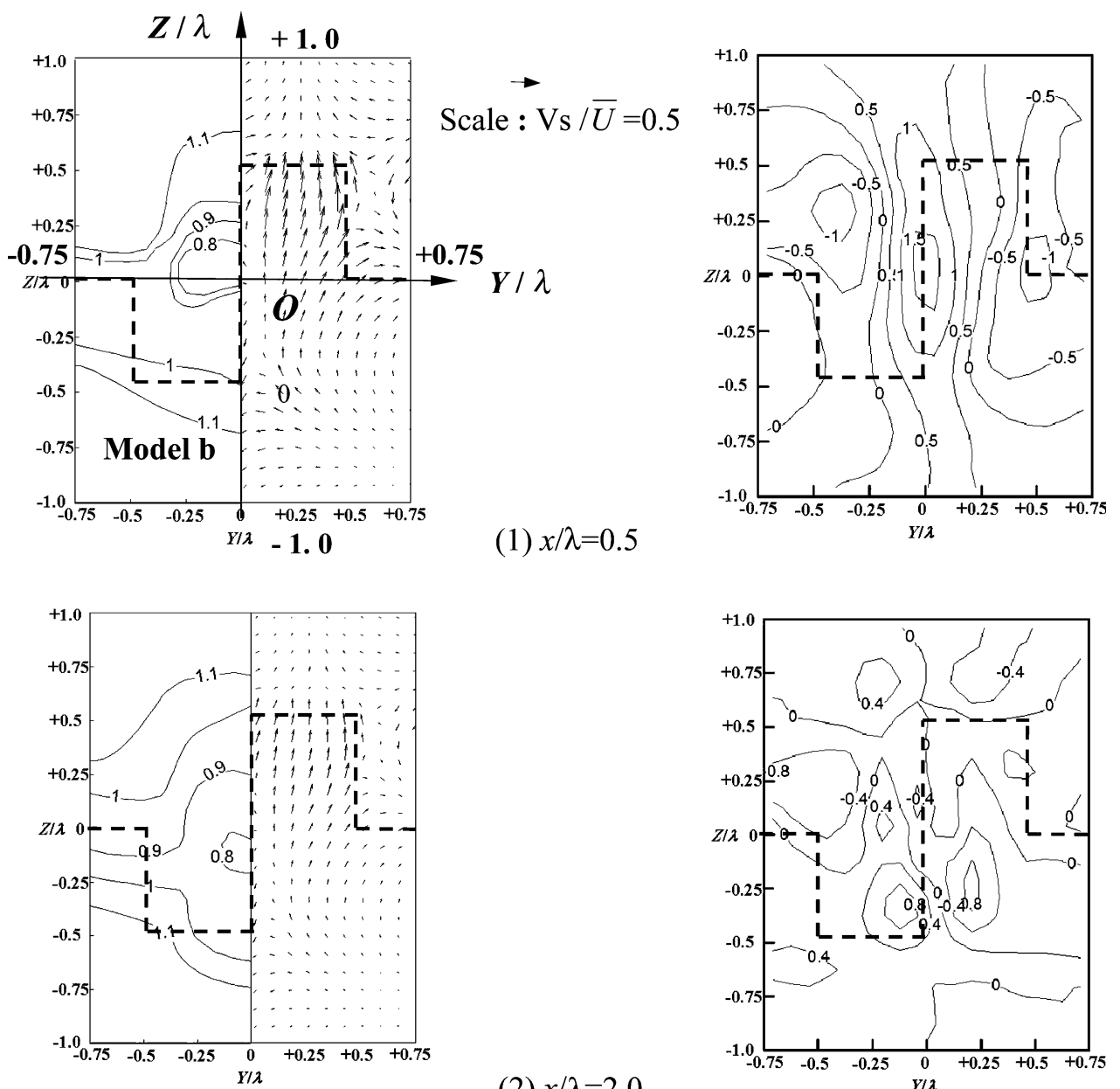

(2) $x / \lambda=2.0$
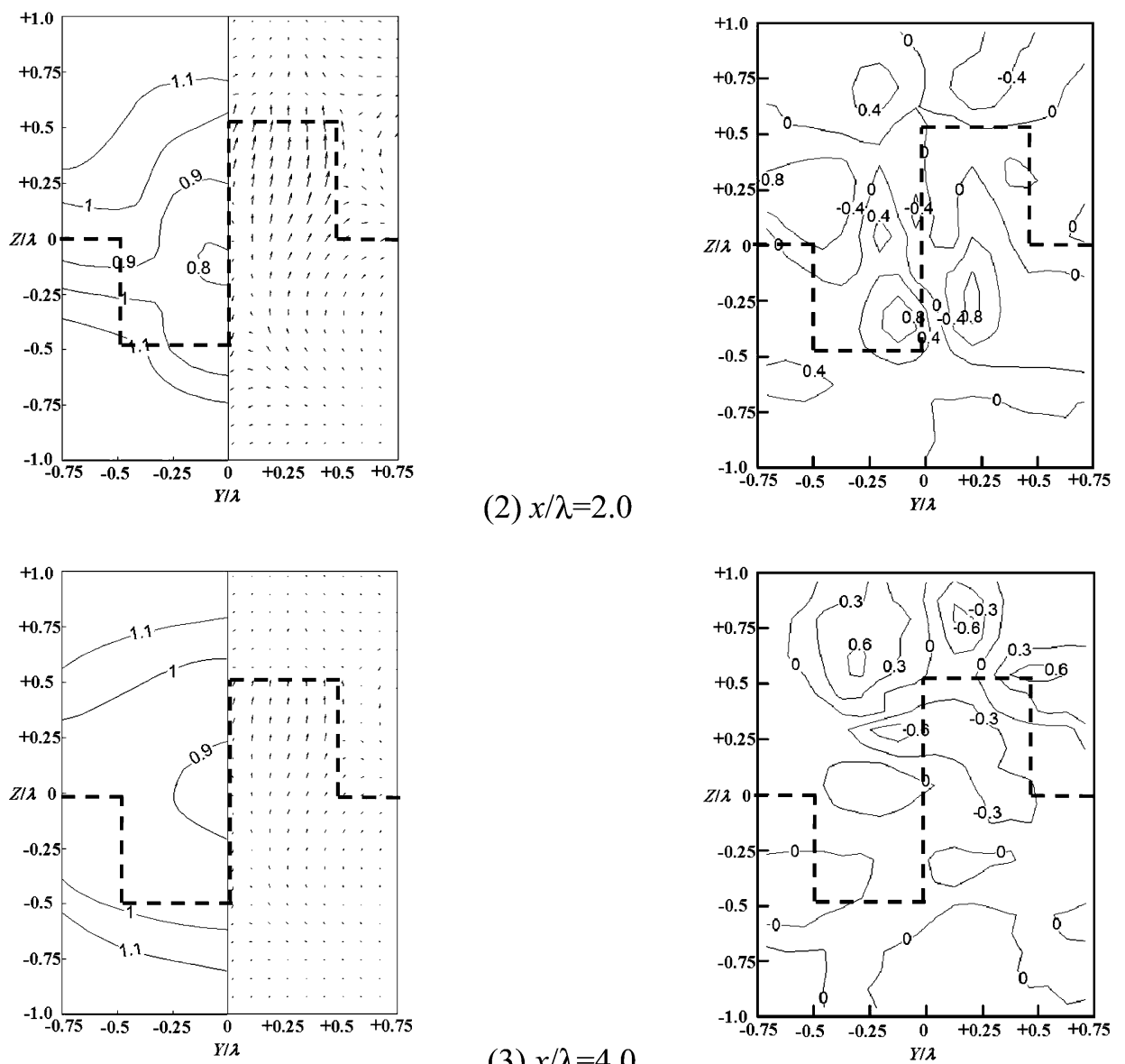

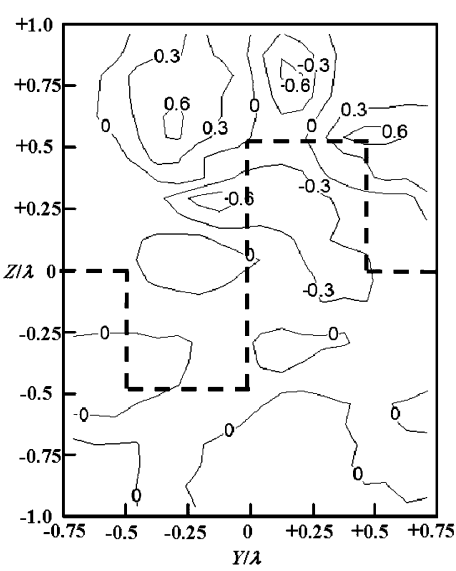

Figure 17 shows the variation of the normalized streamwise circulation with downstream distance of different modified configurations, i.e., the scalloped model $\mathrm{d}$ and scarfed model e, for both velocity ratios $r=1$ and 0.4 . Results of the unmodified basic model a were also shown for comparison. It could be observed that the relative magnitudes and variation trends for the three models were almost the same for both velocity ratios. All of them decayed exponentially with downstream distance, and reached negligible magnitude within the near field of the wake region at $x / \lambda=6$. However, their initial circulations and the decay rates were somewhat different. For the scalloped model d, its initial circulation was of the highest magnitude, which were about $12 \%$ higher than the basic model a. The reason had been explained earlier, which was mainly due to additional streamwise vortices formed near 
Fig. 14 Contours of $(U / \bar{U})$, the secondary flow velocity vectors $\left(\overrightarrow{V_{\mathrm{s}}} / \bar{U}\right)$ and streamwise vorticity contours at different downstream stations after the trailing edge of scalloped-lobe forced mixer (model d) for velocity ratio $r=0.4$
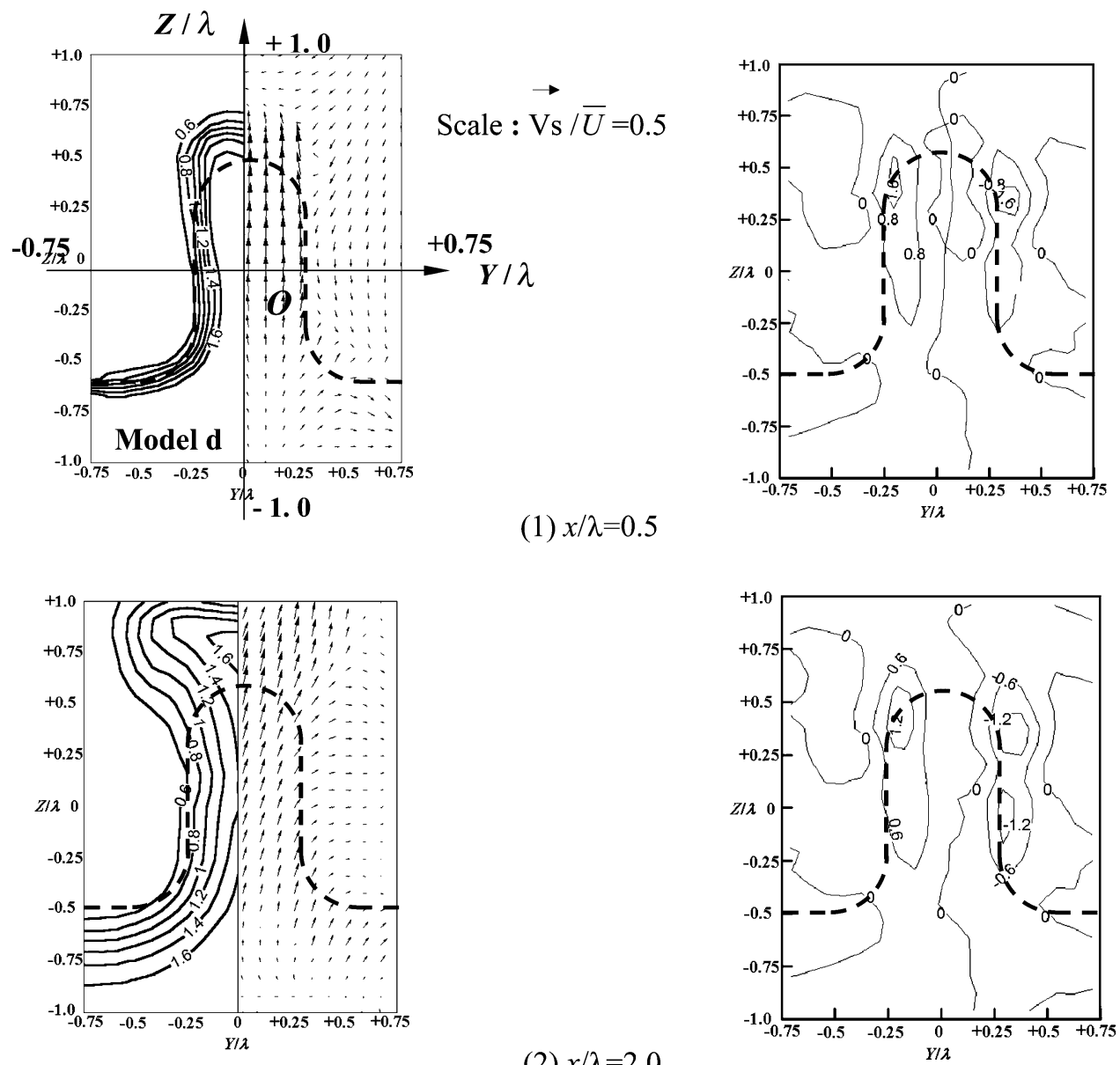

(2) $x / \lambda=2.0$

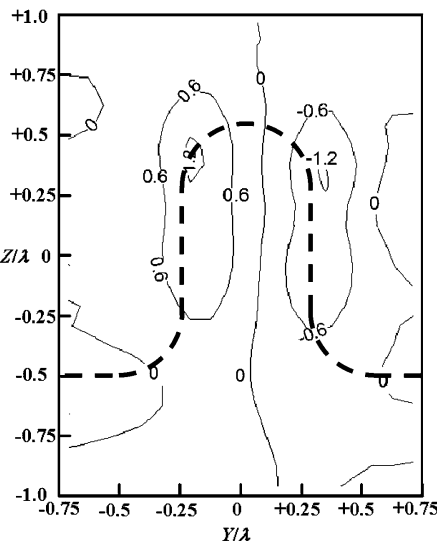

the notches. These additional vortices strengthened the magnitude of the total streamwise vortices. Meanwhile, it could be seen from the figure that the streamwise vorticity would breakdown faster for the scalloped mixer than the basic model a. Similar phenomenon has been observed in the serial experiments of the multi-lobe forced mixers by $\mathrm{Yu}$ et al. (1995). The faster streamwise vorticity-decaying rate of the scalloped mixer should be imputed to the vortex merging among the main streamwise vortex and the other two additional streamwise vortices. As far as it is known, the vortex merging between like signed vortices would weaken the vorticity of the resulting vortex due to its deformation and filamentation (Waugh 1992). For the scarfed model e, the streamwise circulation generated in its wake was about $75 \%$ of the basic unmodified model a only because of its shortened axial length of the penetration region. To shorten the axial length means to reduce the lobe height as well. The lobe height at its lower side is 


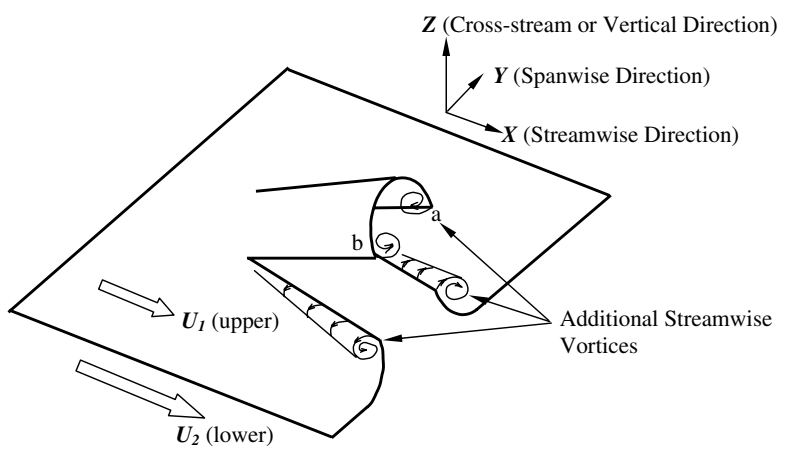

(a) Perspective view

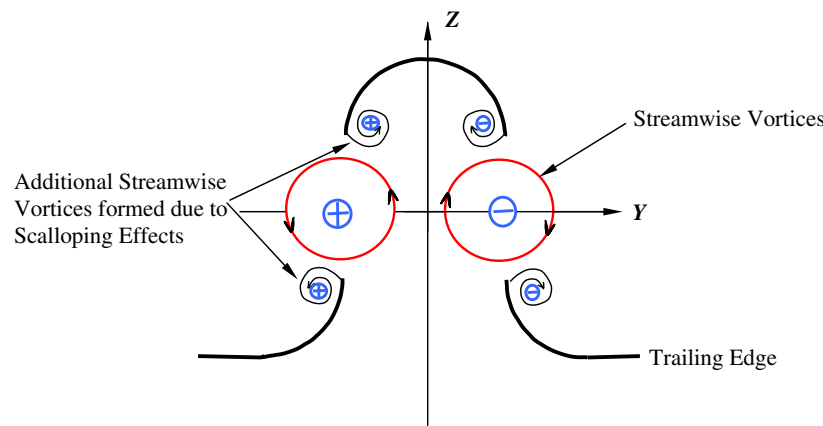

(b) End view (vortices are merging out of paper)

Fig. 15 Schematic of the vortices in the vicinity of the trailing edge of the scalloped-lobe forced mixer (model d)

about $16.9 \mathrm{~mm}$ only as shown in Fig. 2e(1). It was proved that the streamwise vorticity is in direct proportion to the lobe height (Mao et al. 2006). So the streamwise vorticity in the wake of scarfed model would be inferior to the basic model a.

\section{Mixing indicators}

\subsection{Momentum thickness}

The shear layer entrainment momentum thickness in the mixing layer is defined as:

$\theta_{\mathrm{m}}=\int_{-\infty}^{+\infty} \frac{\left(U_{1}-u\right) \times\left(u-U_{2}\right)}{\left(U_{1}-U_{2}\right)^{2}} \times \mathrm{d} z$,

in which $U_{1}$ and $U_{2}$ are the velocities of the on-coming upper and lower streams, while $u$ is the mean streamwise velocity averaged from $y=-45 \mathrm{~mm}$ to $+45 \mathrm{~mm}$, and $Z$ is the lateral direction.

Figure 18 shows the variations of the momentum thickness in the mixing layer of the mixers with different configurations at velocity ratio $r=0.4$. It is clear that the momentum thickness grew very fast within the initial two wavelengths after the trailing edge. At $x / \lambda \geq 3$ the growth rate of the momentum thickness slowed down. The rectangular model $\mathrm{b}$ had higher momentum thickness than model a, which was mainly due to the fact that the mean streamwise vorticity for the rectangular model is higher. The momentum thickness in the wake of triangular model c was the lowest. This result was in accordance with the former facts on its $\mathrm{K}-\mathrm{H}$ and streamwise vortices. Its periodical $\mathrm{K}-\mathrm{H}$ vortices were shed at the lowest Strouhal number; and its mean streamwise vorticity was the least among the three models. The mixing performance of the triangular model $\mathrm{c}$ was the lowest. The scalloped model $\mathrm{d}$ had the highest initial momentum thickness, and grew rapidly from the station $x / \lambda=0.5-1.0$. Both of these two
Fig. 16 Variation of the streamwise circulation with downstream distance of models $\mathrm{a}, \mathrm{b}$ and $\mathrm{c}(r=1$ and 0.4$)$

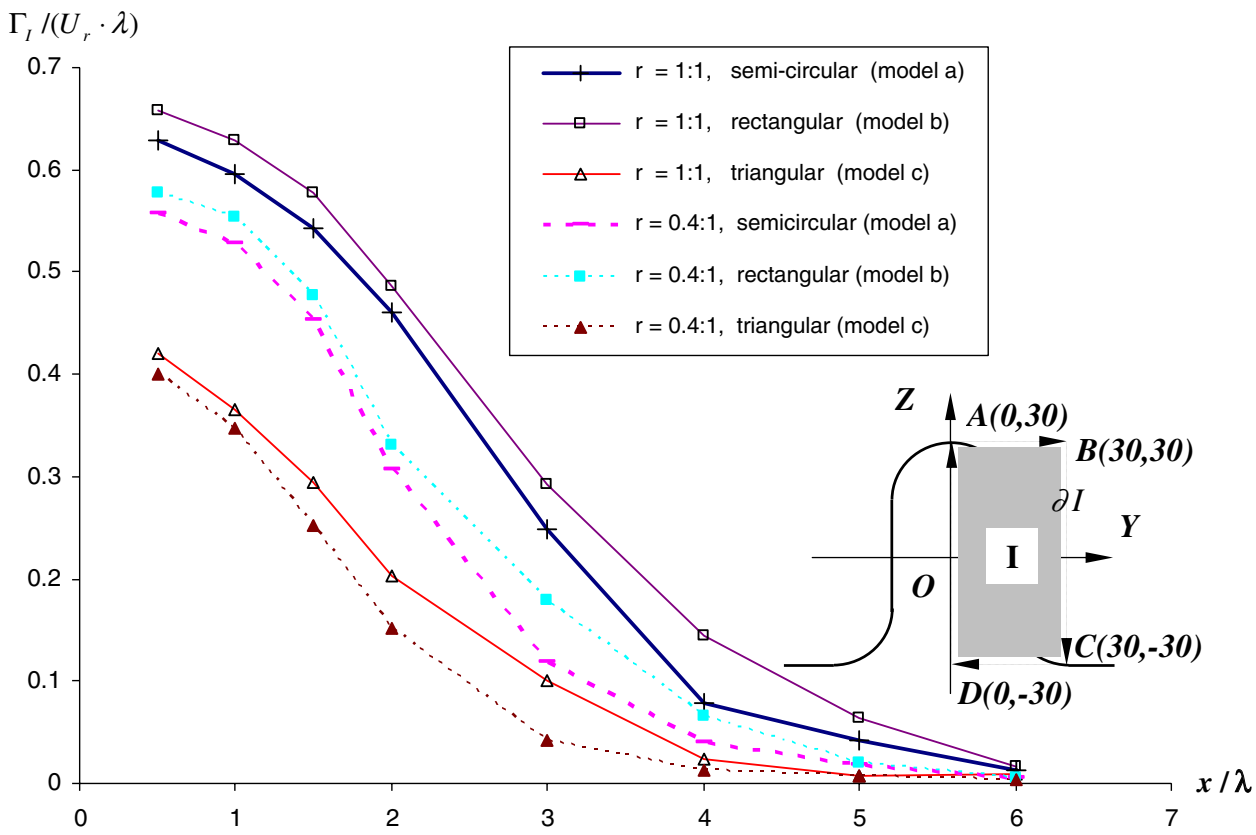


Fig. 17 Variation of the streamwise circulation with downstream distance of models $\mathrm{a}, \mathrm{d}$ and $\mathrm{e}(r=1$ and 0.4$)$
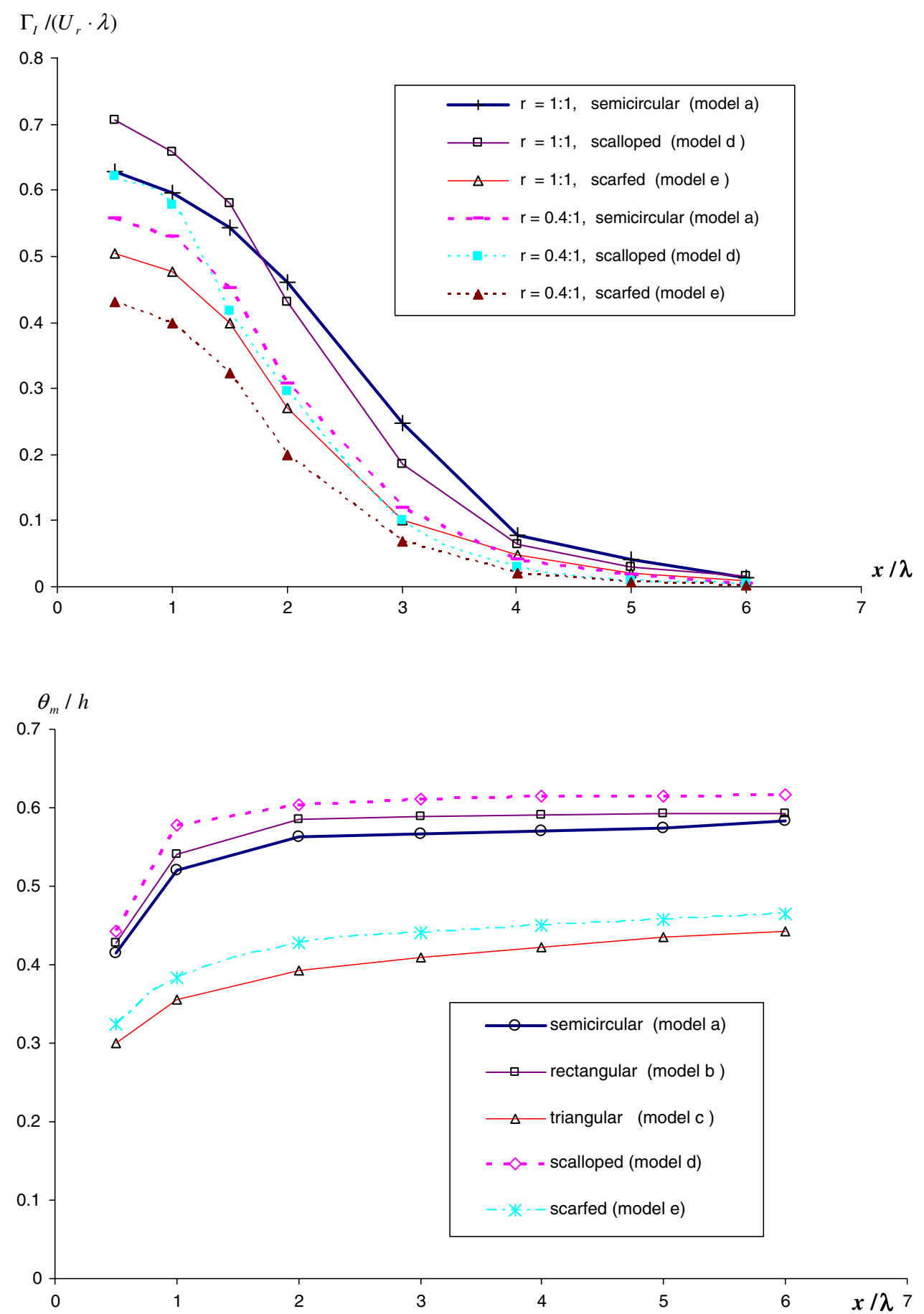

2
Fig. 18 Variation of the momentum thickness for all models in the streamwise direction $r=0.4$
In the absence of any external force, e.g., friction, the momentum is conserved. For the mixer configurations that we had considered, the momentum thickness at the wake would also be linked to the drag induced by the trailing edge geometry. This may explain the difference in the values obtained immediately after the trailing edge for various configurations even under the same upstream conditions. The turbulence levels, low pressure, the streamwise the three different modified test models. 
Fig. 19 Variation of shape factor for all models $r=0.4$

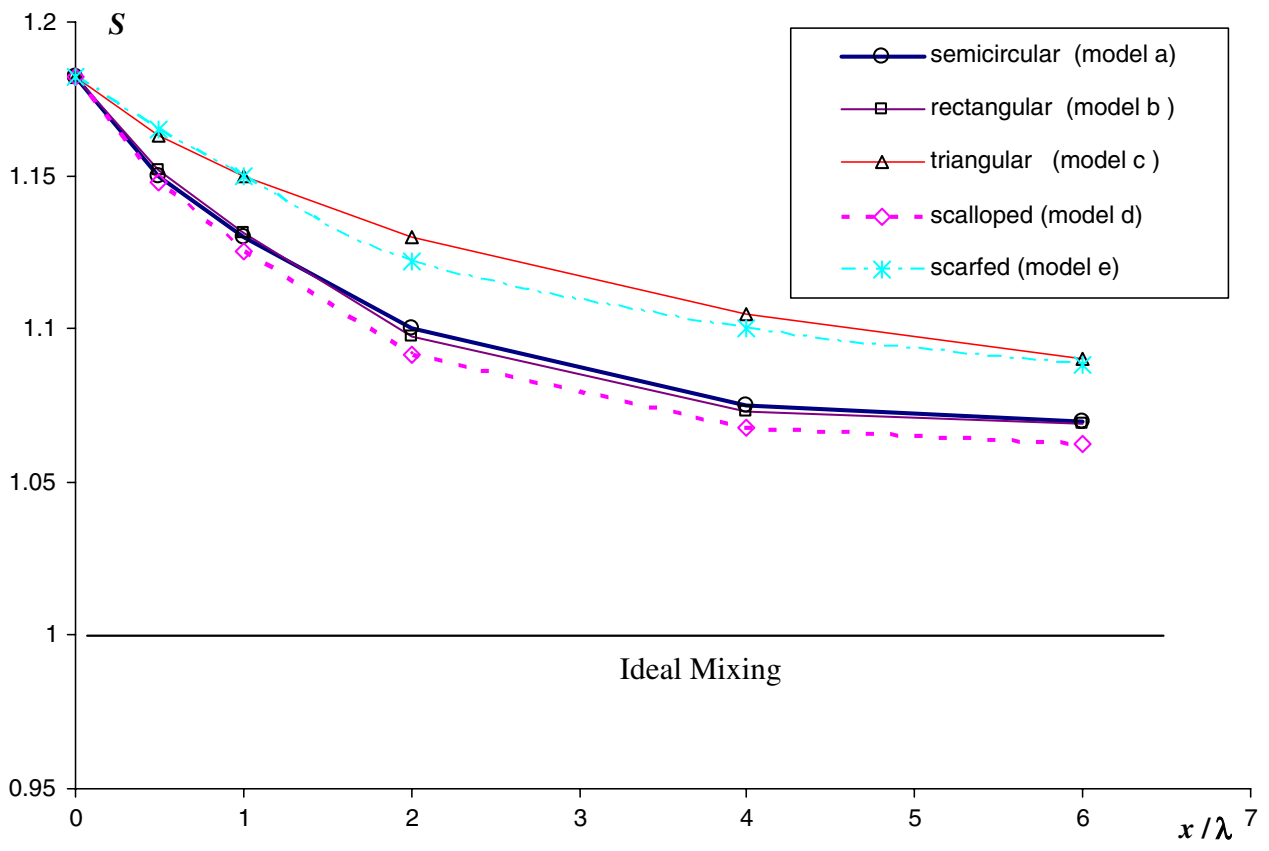

vorticity generated will also affect the subsequent development on mixing performance to far downstream (Antonia and Rajagopalan 1990). This can be seen at the last station of measurements that the momentum thicknesses for respective cases remain different in magnitude.

\subsubsection{Shape factor}

The mixing of the two flows within the wake region in terms of momentum distribution is of interest. Therefore it may be sufficient to define mixedness parameter for the two streams in terms of the shape factor of the streamwise mean velocity distribution, i.e.,

$S=\frac{1}{A_{\text {wake }}} \int_{\text {wake }}\left(\frac{u}{\bar{U}}\right)^{2} \times \mathrm{d} A$,

in which the wake area ended at the border beyond which the velocity difference between the measured values and the nominal velocity $U_{\propto}$ was less than $3 \%$. The definition used here is similar to that used by Bevilaqua (1978) to evaluate the thrust distribution. Ideally, at the location where the two streams at the wake region with different velocities were to be completely mixed, and spatial uniformity was achieved, the momentum distribution would be uniform and the shape factor should be equal to unity. The variation of the shape factor as a function of the downstream distance should provide a useful indication of the extent of the spatial uniformity that the two streams could achieve. Unfortunately for the velocity-matched case $(r=1)$, the shape factor variation was so small that its uncertainty (magnitude at about $\sim 0.28 \%$ ) would conceal its variation. Actually the maximum deviation of its variation was only about $0.5-0.8 \%$ from the ideal mixed condition for this case, which was too small to quantify its actual difference. So only the shape factor for the case at velocity ratio $r=0.4$ would be shown in the present investigation.

It could be observed from Fig. 19 that the shape factor of the basic model a was decreasing along the downstream locations, which implied the ongoing of the mixing progress. The variation of the shape factor for the rectangular mixer $b$ was very close to that of the basic model a. This was in accordance with the magnitudes of their $\mathrm{K}-\mathrm{H}$ and streamwise vortices. As shown earlier, the intensity of the $\mathrm{K}-\mathrm{H}$ vortices for the rectangular mixer was weaker than those of the basic semi-circular mixer. On the other hand, however, the streamwise vorticity of the rectangular mixer was about 5\% stronger than the basic model a. The overall mixing performances of these two mixers are very similar since the $\mathrm{K}-\mathrm{H}$ and streamwise vortices are both contributive to the mixing performance. However, for the triangular mixer, it has been shown that neither its $\mathrm{K}-\mathrm{H}$ vortices nor streamwise vortices were strong. As expected, its mixing performance would be lower than the basic model a indicated by the higher shape factors.

For the other two modified mixers $\mathrm{d}$ and e, their $\mathrm{K}-\mathrm{H}$ vortices were almost of the same intensity as the basic model a. Nevertheless, their streamwise vortices were different. For the scalloped mixer, because higher streamwise circulation would be formed in its wake region, its mixing performance was expected to be better than that of the basic model a. Whereas for the scarfed mixer, its 
mixing performance was likely to be inferior to the basic model a due to its weaker streamwise vorticity. It could be observed from the same figure that the shape factor of the scalloped mixer was a little bit lower than that of the basic model a, implying that the mixing shear layer of the scalloped mixer was more uniform than that of the basic model a. Anyway the difference between these two mixers was not very significant. The shape factor of the scarfed mixer was considerably higher than that of the basic model a, indicating that its mixing performance was much inferior to that of the basic model a. To achieve the same uniformity level for the mixing streams as the basic model a, the scarfed mixer requires more mixing distance.

\section{Conclusions}

Single-lobe forced mixers with three different geometries, i.e., semicircular-lobe, rectangular-lobe and triangular-lobe trailing edges, together with two modified configurations, i.e., the scalloped-lobe and scarfed-lobe configurations, have been examined on their $\mathrm{K}-\mathrm{H}$ vortices and streamwise vortices, respectively. It was found that there were two main frequencies for the Kelvin-Helmholtz vortex in the wake of the forced mixers. The Strouhal numbers would approach their respective maximum values at high Reynolds number. The following conclusions could be drawn based on the present study.

1. The frequencies of the $\mathrm{K}-\mathrm{H}$ vortices shed after the rectangular and triangular mixers are smaller than the basic semicircular mixer. Neither the scalloping nor the scarfing effects would change the frequency of the $\mathrm{K}-\mathrm{H}$ vortices significantly.

2. The rectangular model can generate $5 \%$ higher mean streamwise vorticity than the basic semicircular model whereas the triangular model can only generate $60 \%$ mean streamwise vorticity of the basic semicircular model.

3. The scalloped mixer generates $12 \%$ higher initial mean streamwise vorticity, which decays faster than the basic model whereas the scarfed mixer generates about $75 \%$ mean streamwise vorticity of the basic model only. The interactions between the neighboring streamwise vortices would strengthen the total streamwise vorticity.

4. For velocity ratio at $r=0.4$, the penetration region affected the values of the momentum thickness immediately after the trailing edge of respective mixers such that they may not be comparable directly between different models. Nevertheless, it is noted that the subsequent growth rate of the momentum thickness is similar for the semicircular mixer and the rectangular mixer, which is faster than the triangular mixer. This faster growth rate is influenced directly by the corresponding higher frequency of the $\mathrm{K}-\mathrm{H}$ vortices and the strength of the streamwise vortices. The growth rate for the semicircular model is faster than the scarfed model. However, both of them are still lower than that of the scalloped model, indicating that scalloping improves the mixing performance.

Parameters that are important in determining the mixing performance of individual mixer are (1) Kelvin-Helmholtz vortex, (2) streamwise vortex, and (3) velocity ratio across the splitter plate. These three factors are not independent of each other. For example, criteria (1) and (2) depend both on the geometry of the trailing edge. Factor (3) velocity ratio has more effects on the Kelvin-Helmholtz vortex than the streamwise vortex. Strength of the streamwise vortex, however, did not vary significantly at different velocity ratio (also see $\mathrm{Yu}$ et al. 1995) for one particular mixer trailing edge geometry. When one considers mixing performance for a given flow condition, i.e., at a fixed velocity ratio, it is noted that mixer geometry with parallel side walls (e.g., the semicircular and rectangular mixers) tend to generate stronger streamwise vortex and hence better mixing performance while Kelvin-Helmholtz vortex would have similar strength for respective mixer (see Table 3). Scalloping the lobe may enhance the streamwise vortex by a small margin while scarfing the lobe shows no improvement in enhancing the streamwise vortex strength.

\section{References}

Antonia RA, Rajagopalan S (1990) A comment on the determination of drag of a circular cylinder. AIAA J 28:1833-1835

Barber T, Paterson RW, Skebe SA (1988) Turbofan forced mixer lobe flow modeling, part 1: experimental and analytical assessment, NASA contractor report 4147

Bevilaqua PM (1978) Evaluation of hypermixing for thrust augmenting ejector. J Aerocraft 11(6):348-354

Cheng L, Zhou Y (2008) Surface perturbation technique for flowinduced vibration and noise control. J Sound Vib 310:527-540

Crouch RW, Coughlin CL, Paynter GC (1976) Nozzle exit flow profile shaping for jet noise reduction, AIAA paper, pp 76-511

Durst F, Melling A, Whitelaw JH (1981) Principals and practice of laser-Doppler anemometry. Academic Press, London

Eckerle WA, Sheibani H, Awad J (1992) Experimental measurement of the vortex development downstream of a lobed forced mixer. ASME J Eng Gas Turbines Power 114:63-71

Kuchar AP, Chamberlin R (1980) Scale model performance test investigation of exhaust system mixers for an energy efficiency engine $\left(\mathrm{E}^{3}\right)$ propulsion system, AIAA paper, pp 80-229

Mao R (2005) Two-stream mixing flow with streamwise vorticity, $\mathrm{PhD}$ thesis, Nanyang Technological University, Singapore

Mao R, Yu SCM, Chua LP (2006) Kelvin-Helmholtz and streamwise vortices in the near wake of a single-lobe forced mixer. J Aero Eng Proc Inst Mech Eng Part G 220:279-298 
McCormick DC, Bennett JC Jr (1994) Vortical and turbulent structure of a lobed mixer free shear layer. AIAA J 32(9):1852-1859

Moffat RJ (1988) Describing the uncertainties in experimental results. Exp Therm Fluid Sci 1:3-17

O'Sullivan MN, Krasnodebski JK, Waitz IA, Greitzer EM, Tan CS, Dawes WN (1996) Computational study of viscous effects on lobed mixer flow features and performance. AIAA J Prop Power 12(3):449

Paterson RW (1982) Turbofan forced mixer-nozzle internal flowfield. In: A benchmark experimental study, vol 1, NASA contractor report 3492

Povinelli LA, Anderson BH and Gerstenmaier W (1980) Computation of three dimensional flow in turbofan mixers and comparison with experimental data, AIAA paper, pp 80-227

Roshko A (1955) On the wake and drag of bluff bodies. J Aero Sci 22:124-135

Shumpert PK (1980) An experimental model investigation of turbofan engine internal exhaust gas mixer configurations, AIAA paper, pp $80-228$
Skebe SA, McCormick DC, Presz WM (1988) Parameter effects on mixer-ejector pumping performance, AIAA paper, pp 88-188

Ukeiley L, Glauser M, Wick D (1993) Downstream evolution of proper orthogonal decomposition eigenfunctions in a lobed forced mixer. AIAA J 31(8):1392-1397

Waugh D (1992) The efficiency of symmetric vortex merger. Phys Fluids A 4:1745-1758

Yanta WJ, Smith RA (1973) Measurements of turbulence transport properties with a laser-Doppler velocimeter, AIAA paper, pp 73169

Yu SCM, Xu XG (1998) Flow characteristics of confined coaxial nozzle flow with a central lobed mixer at different velocity ratios. AIAA J 36(3):349-358

Yu SCM, Xu XG, Yip TH (1995) Effects of initial boundary layers to the lobed mixer trailing Streamwise vorticity. AIAA J Prop Power 12(2):440-442

Yu SCM, Hou YX, Chan WK (2000) Effects of scalloping and scarfing to the mixing performances of lobed forced mixers. AIAA J Prop Power 16(3):440-448 